\title{
Control-sharing and merging control Lyapunov functions
}

\author{
Sergio Grammatico, Franco Blanchini, Andrea Caiti
}

\begin{abstract}
Given two control Lyapunov functions (CLFs), a "merging" is a new CLF whose gradient is a positive combination of the gradients of the two parents CLFs. The merging function is an important trade-off since this new function may, for instance, approximate one of the two parents functions close to the origin, while being close to the other far away. For nonlinear control-affine systems, some equivalence properties are shown between the control-sharing property, i.e. the existence of a single control law which makes simultaneously negative the Lyapunov derivatives of the two given CLFs, and the existence of merging CLFs. It is shown that, even for linear time-invariant systems, the control-sharing property does not always hold, with the remarkable exception of planar systems. The class of linear differential inclusions is also discussed and similar equivalence results are presented. For this class of systems, linear matrix inequalities conditions are provided to guarantee the controlsharing property. Finally, a constructive procedure, based on the recently-considered "R-functions", is defined to merge two smooth positively homogeneous CLFs.
\end{abstract}

Index Terms-Composite control Lyapunov functions; stabilizability of linear differential inclusions.

\section{INTRODUCTION}

Control design must quite often compromise among performance, robustness and constraints, and Lyapunov theory offers suitable tools in this regard. The essential goals of the constrained robust performance control design are assuring stability, fulfilling constraints and facing uncertainties. Lyapunovbased techniques for constrained robust control trace back to the 70s [1]. The solutions originally proposed were based on quadratic Lyapunov functions [2] and linear (possibly saturated) controllers. However it became immediately clear that quadratic functions are quite conservative in terms of both domain of attraction (DoA) [3], [4] and robustness margin [5]. Solutions based on non-quadratic Lyapunov functions have been suggested for constrained control, initially based on the polyhedral ones [3], [4] or smoothed-polyhedral functions [6]. An intensive research activity has then been devoted in discovering suitable classes of Lyapunov functions, including the composite Lyapunov functions [7], truncated quadratic functions [8], [9], [10] and polynomial homogeneous functions [11], [12]. Surveys can be found in [13], [14].

There is a fundamental issue in the Lyapunov-based approach for control in which constraints, robustness and optimality are of concern: it turns out that a single Lyapunov

S. Grammatico (grammaticoecontrol.ee.ethz.ch) is with the Automatic Control Laboratory, ETH Zurich, Switzerland. F. Blanchini (blanchini@uniud.it) is with the Department of Mathematics and Informatics, University of Udine, Italy. A. Caiti (a.caiti@dsea.unipi.it) is with the Department of Information Engineering, University of Pisa, Italy. function is typically suitable for one of these goals, but often ineffective for the others. For instance the size of the "safe set", namely the domain of initial conditions for which the constraints are not violated, can be quite large if we consider a particular Lyapunov function. On the contrary, a different Lyapunov function based on some "optimal" cost function and assuring local "optimality", may provide a significantly smaller domain. The established solution to this problem is the control switching strategy. Two controllers are designed, each associated with one of these functions, whose domains of attractions are typically (not necessarily) nested. The control system switches from the "external" to the locally optimal gain as long as the state reaches the "smaller" region of attraction. Obviously, several control gains can be considered with several controlled-invariant regions [15], [16].

The drawback of the scheme is the discontinuity which can be "dangerous", since the system state and the control could be subject to jumps which can be even be persistent in the presence of noise. Therefore it is of interest to find ways to "merge" the two control Lyapunov functions in order to have a "smooth" transient from the level set of the "external" one to the "internal" one. We refer to a procedure of this kind as merging.

Andrieu and Prieur [17], [18] proved that it is possible to merge two Control Lyapunov Functions (CLFs), in a setting actually related to the problem of uniting local and global controllers [19], [20], also addressed in [21] for constrained linear systems. Their technique works under the assumption that there exists a suitable domain in which the two control Lyapunov function share a common control [18, Proposition 2.2]. More recently, Clarke [22] showed how to solve the problem of merging two semiconcave (continuous, locally Lipschitz, but not everywhere-differentiable) CLFs, deriving a semiconcave function based on the min operator.

In this paper, inspired by the mentioned works [17], [18], we investigate the control-sharing property, namely the existence of a single control law which makes simultaneously negative the Lyapunov derivatives of two given Lyapunov functions. We show some equivalence properties between the control sharing and the possibility of adopting a merging procedure.

The control-sharing property is not necessarily satisfied even for linear systems, with the remarkable exception of the planar case (i.e. with two-dimensional state space). Therefore, we provide efficient computational tests to check the controlsharing property for some special classes of functions including polyhedral, quadratic, piecewise quadratic, truncated ellipsoids, and combinations of these ones.

Finally we provide as merging example the technique based 
on the "R-functions" theory, first presented in [23], [24], and we show how local optimality can be compromised with a large DoA, under constraints, adopting a single smooth function.

The essential results of the paper are summarized next.

- For planar linear time-invariant systems two convex CLFs always share a control. A third-order counterexample shows that this is not true in general.

- Given two CLFs $V_{1}, V_{2}$, a merging function $V$ is defined as any positive definite function whose gradient has the form $\nabla V(x)=\gamma_{1}(x) \nabla V_{1}(x)+\gamma_{2}(x) \nabla V_{2}(x)$, where $\gamma_{1}, \gamma_{2}: \mathbb{R}^{n} \rightarrow \mathbb{R}_{>0}$ are continuous functions. For the class of nonlinear control-affine systems, it is shown that any merging function $V$ (i.e. for any possible $\gamma_{1}$ and $\gamma_{2}$ ) is also a CLF if and only if $V_{1}$ and $V_{2}$ share a stabilizing control.

- For the class of linear systems, the above statements are also equivalent to the existence of a "regular" type merging, namely, the case in which $\nabla V$ is "close" to $\nabla V_{1}$ far from the state-space origin and $\nabla V$ is "close" to $\nabla V_{2}$ in a neighborhood of the origin.

- Several conditions are provided to check the controlsharing property. These are based on Linear Programming (LP) in the case of piecewise-linear functions and Linear Matrix Inequalities (LMIs) in the case of piecewisequadratic and truncated-ellipsoidal functions.

- The "R-composition" merging technique presented in [25] is considered to solve the problem of preserving the large DoA under constraints of one CLF and assuring local optimality guaranteed by the other at the same time.

\section{A. Notation}

$I_{n}$ denotes the $n \times n$ identity matrix. $\underline{1}_{s}:=(1,1, \ldots, 1)^{\top} \in$ $\mathbb{R}^{s}$ and $\underline{0}_{s}:=(0,0, \ldots, 0)^{\top} \in \mathbb{R}^{s}$. The notation $\operatorname{co}(\cdot)$ denotes the convex hull. int $\mathcal{S}$ denotes the interior of a set $\mathcal{S}$ and $\partial \mathcal{S}$ denotes its boundary. For any positive (semi)definite function $V: \mathbb{R}^{n} \rightarrow \mathbb{R}_{>0}, \mathcal{L}_{V}$ denotes its 1-level set, i.e. $\mathcal{L}_{V}:=\{x \in$ $\left.\mathbb{R}^{n} \mid V(x) \leq 1\right\}$. Hence, for $\sigma \in \mathbb{R}_{\geq 0}, \mathcal{L}_{(V / \sigma)}:=\left\{x \in \mathbb{R}^{n} \mid\right.$ $V(x) \leq \sigma\}$. A square matrix $W \in \mathbb{R}^{s \times s}$ is an $\mathcal{M}$-matrix if $W_{i, j} \geq 0 \forall i \neq j$. For any couple of vectors $x, y \in \mathbb{R}^{n}$, the inequality $x \leq y$ denotes the componentwise inequalities $x_{i} \leq y_{i}$ for all $i=1, \ldots, N$.

\section{TECHNICAL BACKGROUND AND NEGATIVE RESULTS}

Let us consider nonlinear control-affine systems

$$
\dot{x}=f(x)+g(x) u,
$$

where $x \in \mathbb{R}^{n}$ is the state variable, $u \in \mathbb{R}^{m}$ is the control input, and $f: \mathbb{R}^{n} \rightarrow \mathbb{R}^{n}$, with $f(0)=0, g: \mathbb{R}^{n} \rightarrow \mathbb{R}^{n \times m}$ are locally-bounded functions. We also consider the following notion of control Lyapunov function.

Definition 1 (Control Lyapunov Function). A positive definite, radially unbounded, smooth away from zero, function $V: \mathbb{R}^{n} \rightarrow \mathbb{R}_{\geq 0}$ is a control Lyapunov function for (1) if there exists a locally-bounded control law $u: \mathbb{R}^{n} \rightarrow \mathbb{R}^{m}$ such that for all $x \in \mathbb{R}^{n}$ we have

$$
\nabla V(x)(f(x)+g(x) u(x))<0 .
$$

$V$ is a control Lyapunov function with domain $\mathcal{L}_{(V / \sigma)}$, for $\sigma>0$, if (2) holds for all $x \in \mathcal{L}_{(V / \sigma)}$.

The following definition is fundamental in the sequel.

Definition 2 (Control-Sharing Property). Two control Lyapunov functions $V_{1}$ and $V_{2}$ for (1) have the controlsharing property if there exists a locally-bounded control law $u: \mathbb{R}^{n} \rightarrow \mathbb{R}^{m}$ such that for all $x \in \mathbb{R}^{n}$ we have the following inequalities simultaneously satisfied.

$$
\begin{aligned}
& \nabla V_{1}(x)(f(x)+g(x) u(x))<0 \\
& \nabla V_{2}(x)(f(x)+g(x) u(x))<0
\end{aligned}
$$

$V_{1}$ and $V_{2}$ have the control-sharing property under constraints $x \in \mathbb{X} \subseteq \mathbb{R}^{n}, u \in \mathbb{U} \subseteq \mathbb{R}^{m}$ if (3) holds for all $x \in \mathbb{X}$ with a constrained control law $u: \mathbb{X} \rightarrow \mathbb{U}$.

For the class of control-affine differential inclusions

$$
\dot{x} \in F(x)+G(x) u,
$$

where $F: \mathbb{R}^{n} \rightrightarrows \mathbb{R}^{n}$ and $G: \mathbb{R}^{n} \rightrightarrows \mathbb{R}^{n \times m}$ are compactvalued mappings, the previous definitions hold unchanged provided that conditions (2) and (3) holds with $\dot{x}=\varphi+\Gamma u$, for all $(\varphi, \Gamma) \in(F(x), G(x))$.

\section{A. Negative results on control sharing, even for linear systems}

Let us also consider Linear Time-Invariant (LTI) systems

$$
\dot{x}=A x+B u,
$$

where $x \in \mathbb{R}^{n}, u \in \mathbb{R}^{m}, A \in \mathbb{R}^{n \times n}$ and $B \in \mathbb{R}^{n \times m}$.

For second-order systems, we have the following result on the control-sharing property.

Theorem 1. Two convex CLFs for (5) do necessarily have the control-sharing property if $n \leq 2$.

Remark 1. The previous results extends that provided in [17, Proposition 2], where it is shown that for planar linear systems there always exists a common control law between two quadratic CLFs. Here we show that such a property is valid for convex CLFs of any class.

However, even for second-order systems, the previous result is not "robust". Consider the class of Linear Differential Inclusions (LDIs)

$$
\dot{x} \in \operatorname{co}\left\{A_{i} x+B_{i} u \mid i \in[1, N]\right\},
$$

for some integer $N>0, A_{i} \in \mathbb{R}^{n \times n}$ and $B_{i} \in \mathbb{R}^{n \times m}$ for all $i \in[1, N]$. The result of Theorem 1 does not hold for this class of systems according to the following result.

Proposition 1. Two CLFs for (6) do not necessarily have the control-sharing property.

In general, for $n>2$, the control-sharing property does not hold even for LTI systems.

Proposition 2. Two CLFs for (5) do not necessarily have the control-sharing property if $n>2$. 


\section{Merging control Lyapunov functions}

Throughout the paper, we refer to $V_{1}$ and $V_{2}$ as given CLFs. The class of systems under consideration will be indeed always explictly mentioned.

Standing Assumption 1. Functions $V_{1}, V_{2}: \mathbb{R}^{n} \rightarrow \mathbb{R}_{\geq 0}$ are two CLFs.

\section{A. Gradient-type merging control Lyapunov functions}

Definition 3 (Gradient-type merging CLF). Let $V: \mathbb{R}^{n} \rightarrow$ $\mathbb{R}_{\geq 0}$ be positive definite and smooth away from zero. $V$ is a gradient-type merging candidate if there exist two continuous functions $\gamma_{1}, \gamma_{2}: \mathbb{R}^{n} \rightarrow \mathbb{R}_{\geq 0}$ such that $\left(\gamma_{1}(x), \gamma_{2}(x)\right) \neq$ $(0,0)$ and

$$
\nabla V(x)=\gamma_{1}(x) \nabla V_{1}(x)+\gamma_{2}(x) \nabla V_{2}(x) .
$$

$V$ is a gradient-type merging CLF if, in addition, it is a CLF.

Remark 2. The blending CLF $V(x)=\min \left\{V_{2}(x), c \cdot V_{1}(x)+\right.$ $d\}$ [22, Section 9], for opportune constants $c, d>0$, does not fall into the class of gradient-type merging because it is not a differentiable function. However it can be approximated with arbitrary precision by the "smoothed min" $V=$ $\sqrt[p]{V_{2}^{p}+\left(c \cdot V_{1}+d\right)^{p}}$ for $p<0$ and $|p|$ large enough.

Merging functions form a class much wider of those considered specifically later. For instance, the "smoothed $\max " V:=$ $\sqrt[p]{V_{1}^{p}+V_{2}^{p}}$, for $p>0$, or $V:=\rho_{1}\left(V_{1}, V_{2}\right) V_{1}+\rho_{2}\left(V_{1}, V_{2}\right) V_{2}$ are possible merging candidates.

For nonlinear systems (1), we show that any gradient-type merging candidate is a CLF if and only if there exists a common stabilizing controller between the CLFs $V_{1}$ and $V_{2}$.

Theorem 2. The following statements are equivalent for (1).

1) Any gradient-type merging of $V_{1}$ and $V_{2}$ is a CLF.

2) $V_{1}$ and $V_{2}$ have the control-sharing property.

Remark 3. The main contribution of Theorem 2 relies on the necessity of the existence of a common control law, i.e. implication 1) $\Longrightarrow 2$ ); conversely, the sufficient part, i.e. $2) \Longrightarrow 1$ ) may follow from the results in $[18$, Theorem 1 , Proposition 1]. We also notice that since the system (1) is control-affine, the existence of a stabilizing common control law is equivalent to the existence of a continuous stabilizer, see [22, Theorem 1.5], [26, Section 5.9].

Remark 4. The equivalence result of Theorem 2 can be further exploited to address stabilization under constraints. An interesting setting, very similar to the one of [18], is whenever there exist $r, R>0$, with $0<r<R$, such that $V_{1}$ is CLF in the set $\left\{x \in \mathbb{R}^{n} \mid\|x\| \geq r\right\}$ and $V_{2}$ is CLF in the set $\left\{x \in \mathbb{R}^{n} \mid\|x\| \leq R\right\}$.

\section{B. Regular gradient-type merging}

The property that any gradient-type merging of two CLFs is a CLF is quite strong. In practice we will be interested in the case in which the gradient-type merging candidate $V$ has the same domain of $V_{1}$, namely $\mathcal{L}_{V}=\mathcal{L}_{V_{1}} ; V$ has its gradient $\nabla V(x)$ aligned with $\nabla V_{1}(x)$ whenever $x \in \partial \mathcal{L}_{V}$, while ("almost") aligned with $\nabla V_{2}(x)$ whenever $x$ is "close" to the origin.

Definition 4 (Regular gradient-type merging CLF). A gradient-type merging candidate $V$ is regular with tolerance $\varepsilon \geq 0$ if $\mathcal{L}_{V}=\mathcal{L}_{V_{1}}$ and the associated functions $\gamma_{1}, \gamma_{2}$ satisfy the following conditions.

$$
\begin{gathered}
\left\{\gamma_{1}(x)=1, \gamma_{2}(x)=0\right\} \Longleftrightarrow x \in \partial \mathcal{L}_{V_{1}} ; \\
0 \leq \gamma_{1}(0) \leq \varepsilon, 1-\varepsilon \leq \gamma_{2}(0) \leq 1 .
\end{gathered}
$$

A gradient-type merging candidate $V$ is regular if it is regular with tolerance $\varepsilon=0 . V$ is a regular gradient-type merging CLF if, in addition, it is a CLF.

We then consider regular control laws $u(\cdot)$, namely we consider a "small control property", meaning that $u(x)$ goes to 0 at least linearly as $x$ goes to 0 .

Definition 5 (Regular control). A control law $u: \mathbb{R}^{n} \rightarrow \mathbb{R}^{m}$ is regular if it is continuous and for any given $x \in \mathbb{R}^{n}$ the limit

$$
\bar{u}_{x}:=\lim _{\lambda \rightarrow 0^{+}} \frac{u(\lambda x)}{\lambda}
$$

exists and satisfies $\left\|\bar{u}_{x}\right\|<\infty$.

The meaning is that a control law is regular if it is continuous and "locally homogeneous". For instance, in the case of an homogeneous control $u=\phi(x)$ (hence also linear $u(x)=K x)$, namely such that $\phi(\lambda x)=\lambda \phi(x)$, for all $\lambda \geq 0$, we have $\bar{u}_{x}=\phi(x)$, so that $\left\|\bar{u}_{x}\right\|<\infty$.

For linear systems (5), we have the following result for the regular gradient-type merging.

Theorem 3. Assume that $V_{1}$ and $V_{2}$ are positively homogeneous CLFs of the same degree, each associated with a regular control. Then, the following statements are equivalent for (5).

1) There exists a regular gradient-type merging CLF associated with a regular control.

2) Any gradient-type merging is a CLF associated with a regular control.

3) $V_{1}$ and $V_{2}$ share a regular control.

Remark 5. Assuming positively homogeneous CLFs is a limitation. Choosing the same degree of homogeneity is without loss of generality because, if $\dot{V} \leq-\eta V$, for some $\eta>0$, then $\left(\dot{V}^{p}\right) \leq-\eta p V^{p}$ for any real $p>0$.

We can relate our "regular merging" CLFs to the literature on "blending" CLFs [22] and "uniting" CLFs [18], [20] as follows. In [22, Theorem 9.1], it is shown that from the knowledge of two CLFs $V_{1}, V_{2}$, it is possible to build up a "blending" CLF of the form $V(x)=\min \left\{V_{1}(x), c V_{2}(x)+d\right\}$, for appropriate $c, d \geq 0$, so that $V$ necessarily admits a stabilizing controller $\kappa: \mathbb{R}^{n} \rightarrow \mathbb{R}^{m}$ of the form $\kappa(x) \in$ $\left\{\kappa_{1}(x), \kappa_{2}(x)\right\}$. We show that even for linear systems (5), the result does not necessarily hold for gradient-type merging CLFs, namely because of the differentiability property of gradient-type merging candidates.

Proposition 3. Assume $\kappa_{1}, \kappa_{2}: \mathbb{R}^{n} \rightarrow \mathbb{R}^{m}$ are control laws respectively associated with $V_{1}$ and $V_{2}$. Then, even 
for linear systems (5), a regular gradient-type merging CLF $V$ does not necessarily admit a control law of the kind $\kappa(x) \in\left\{\kappa_{1}(x), \kappa_{2}(x)\right\}$.

Remark 6. For nonlinear control-affine systems, [20, Section 2.2] shows that there exists a topological obstruction in uniting a local and a global controller by means of a static timeinvariant continuous control law. It follows from the proof of Proposition 3, see Appendix A-F, that such a obstruction is also valid for the class of linear systems whenever we look for a controller of the kind used in [22, Proof of Theorem 9.1].

\section{Gradient-type merging for differential inclusions}

We now consider nonlinear differential inclusions (4) and we provide the following results.

Proposition 4. If $V_{1}$ and $V_{2}$ have the control-sharing property for (4), then any gradient-type merging is a CLF.

Theorem 4. Assume that, in (4), the mapping $G$ is singlevalued. Then the following statements are equivalent for (4).

1) Any gradient-type merging is a CLF.

2) $V_{1}$ and $V_{2}$ have the control-sharing property.

The result of Theorem 4 does also apply to LDIs (6) having $B_{i}=B$ for all $i \in[1, N]$.

\section{CONDITIONS FOR THE EXISTENCE OF A COMMON CONTROLLER}

In this section we consider the class of LDIs (6) and we propose several matrix inequality conditions for the existence of a common controller between the CLFs $V_{1}$ and $V_{2}$. For ease of presentation, the matrix conditions presented next do not include the control constraints; however, they can be considered without conceptual difficulties. We address the following classes of homogeneous functions: (symmetric) polyhedral, quadratic, max of quadratics and truncated ellipsoids.

Remark 7. Note that some of the mentioned functions are nonsmooth. However, we can apply the smoothing procedure in [27]. For instance, if $\|F x\|_{\infty}^{2}$ is a polyhedral CLF (PCLF) with a certain control law $\kappa$ for an LDI (6), the same control law $\kappa$ assures that $\|F x\|_{2 p}^{2}$ is a Lyapunov function if $p>0$ is taken large enough [27]. Therefore if the CLF $V_{1}(x)=\|F x\|_{\infty}^{2}$ shares a control with the CLF $V_{2}$, then also $\|F x\|_{2 p}^{2}$ does for $p$ sufficiently large.

Let $V_{p}: \mathbb{R}^{n} \rightarrow \mathbb{R}_{>0}$ be a positive definite polyhedral function and let $X=\left[x_{1}\left|x_{2}\right| \ldots \mid x_{s}\right] \in \mathbb{R}^{n \times s}$ be the matrix whose columns are the vertices of $\mathcal{L}_{V_{p}}$, i.e. [14, Equation (4.28)]

$$
\begin{aligned}
& V_{p}(x):=\min \left\{\underline{1}_{s}^{\top} \alpha \mid x=X \alpha, \alpha \geq \underline{0}_{s}\right\}= \\
& \min \left\{\sum_{j=1}^{s} \alpha_{j} \mid \sum_{j=1}^{s} \alpha_{j} x_{j}=x, \alpha_{j} \geq 0 \forall j \in[1, s]\right\} .
\end{aligned}
$$

The dual version of $(8)$ is $V_{p}(x):=\max _{i \in[1, s]} F_{i} x$ [14, Equation (4.27)], where $F_{i}$ is the $i$ th row of a full column rank matrix $F \in \mathbb{R}^{s \times n}$ and $\mathcal{L}_{V_{p}}:=\left\{x \in \mathbb{R}^{n} \mid F x \leq \underline{1}_{s}\right\}$.
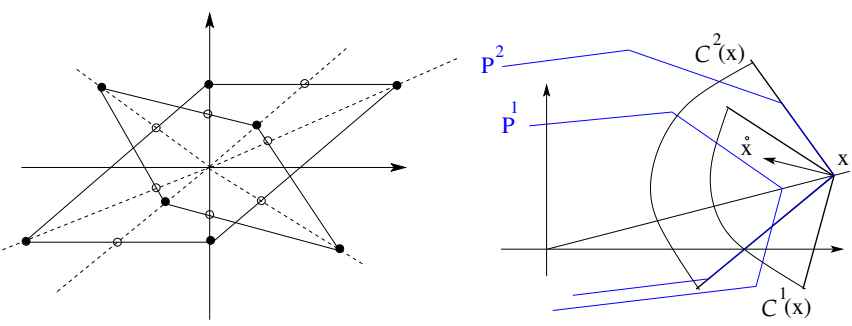

Fig. 1. Left plot: in the construction of Theorem 5 we add redundant vertices for $\mathcal{L}_{V_{1}}$ and $\mathcal{L}_{V_{2}}$, which hence have "true vertices" (black) and "fictitious vertices" (white). Right plot: (scaling-invariant) tangent cones to the polyhedra $P^{1}$ and $P^{2}$. The derivative vector $\dot{x}$ of any point $x$ must be in the intersection of the cones for the control-sharing property to hold true.

Then $V_{p}$ is a PCLF for (6) if and only if there exist $\eta>0$, $\mathcal{M}$-matrices $W_{1}, W_{2}, \ldots, W_{N} \in \mathbb{R}^{s \times s}$ and $U \in \mathbb{R}^{m \times s}$ such that for all $i \in[1, N]$ we have [14, Proposition 7.19]

$$
\begin{aligned}
A_{i} X+B_{i} U & =X W_{i} \\
\underline{1}_{s}^{\top} W_{i} & \leq-\eta \underline{1}_{s}^{\top},
\end{aligned}
$$

which is equivalent to

$$
\left[\eta I+A_{i}\right] X+B_{i} U=X\left[W_{i}+\eta I\right]=X \hat{W}_{i}, \underline{1}_{s}^{\top} \hat{W}_{i} \leq \underline{0}_{s} .
$$

The meaning is that $V_{p}$ is a CLF assuring a decreasing rate $\eta>0$ if and only if $V_{p}$ is non-increasing for the modified system $\dot{x} \in \operatorname{co}\left\{A_{i}^{\eta} x+B_{i} u \mid i \in[1, N]\right\}$, where $A_{i}^{\eta}:=\eta I+A_{i}$.

For any given $x \neq 0$, consider the set of all points $y$ in which $V_{p}(y) \leq V_{p}(x)$, i.e. $\mathcal{L}_{V_{p} / V_{p}(x)}$, and consider the tangent cone $\mathcal{C}(x):=\left\{z \in \mathbb{R}^{n} \mid \exists h>0: x+h z \leq V_{p}(x)\right\}$ (see Figure 1). The tangent cone $\mathcal{C}(x)$ has the properties that it is invariant under positive scaling, $\mathcal{C}(x)=\mathcal{C}(\lambda x)$ for all $\lambda>0$, and that, if we use the dual representation, it is defined by the active constraints, namely $F_{i} x \leq 0$ for all $i$ such that $F_{i} x=V_{p}(x)$.

Then (10) has the following interpretation: for any $x \neq 0$ we have the differential inclusion $\dot{x} \in \operatorname{co}\left\{A_{i}^{\eta} x+B u \mid i \in\right.$ $[1, N]\} \in \mathcal{C}(x)$.

We assume that $V_{1}$ and $V_{2}$ are two PCLFs of the form (8), with matrices of vertices $X^{1}:=\left[x_{1}^{1}|\ldots| x_{s_{1}}^{1}\right]$ and $X^{2}:=$ $\left[x_{1}^{2}|\ldots| x_{s_{2}}^{2}\right]$, respectively. For any $x \neq 0$, we denote by $\mathcal{C}^{1}(x)$ and $\mathcal{C}^{2}(x)$, the tangent cones respectively associated with $V_{1}(x)$ and $V_{2}(x)$.

We then extend matrices $X^{1}$ and $X^{2}$ by adding fictitious vertices in each of them (the empty dots in Figure 1). Precisely, for each column of $X^{1}$, namely each vertex $x_{k}^{1}$, we take point $\tilde{x}_{k}^{1}:=c x_{k}^{1} \in \partial \mathcal{L}_{V_{2}}$, for appropriate $c>0$ (see Figure 1). Analogously, take $\tilde{x}_{k}^{2}:=c x_{k}^{2} \in \partial \mathcal{L}_{V_{1}}$, for appropriate $c>0$. We define the so extended matrices of dimension $\left(n \times\left(s_{1}+\right.\right.$ $\left.\left.s_{2}\right)\right)$ as

$$
\bar{X}^{1}:=\left[X_{1}\left|\tilde{x}_{1}^{2}\right| \ldots \mid \tilde{x}_{s_{2}}^{2}\right], \quad \bar{X}^{2}:=\left[\tilde{x}_{1}^{1}|\ldots| \tilde{x}_{s_{1}}^{1} \mid X_{2}\right] .
$$

These matrices are valid (redundant) verticesrepresentations for $V_{1}$ and $V_{2}$. We can now establish the result that there exists a common control law between $V_{1}$ and $V_{2}$ if and only if for each vector $\bar{x}_{k}^{1}$ of $V_{1}$ we can find a control vector $u_{k}$ which "decreases" both $V_{1}$ and $V_{2}$.

Theorem 5. $V_{1}$ and $V_{2}$ have the control-sharing property if and only if for each column $\bar{x}_{k}^{1}$ of $\bar{X}^{1}$ (or equivalently of $\bar{X}^{2}$ ), 
which is the representation of $V_{1}\left(V_{2}\right)$ defined in (11), there exist $\eta>0$ and $u_{k} \in \mathbb{R}^{m}$, such that

$$
\left[\eta I+A_{i}\right] \bar{x}_{k}^{1}+B_{i} u_{k} \in \mathcal{C}^{1}\left(\bar{x}_{k}^{1}\right) \bigcap \mathcal{C}^{2}\left(\bar{x}_{k}^{1}\right) .
$$

Since the tangent cones can be represented via linear inequalities, the condition of the theorem requires linear programming.

We now consider the control-sharing between polyhedral and quadratic CLF (QCLF) for (6).

Theorem 6. Assume that $V_{1}=V_{p}$ as in (8) and $V_{2}(x)=$ $x^{\top} P x$ respectively are PCLF and QCLF for (6). Let $r$ be the number of facets of $\mathcal{L}_{V_{1}}$ and let $\mathcal{V}_{k}$ be the set of the vertices belonging to the kth facet, whose cardinality is $s_{k} \in[1, s]$. For all $k \in[1, r]$ and $i \in[1, N]$, define the matrices $S_{k, i}(\eta, U) \in$ $\mathbb{R}^{s_{k} \times s_{k}}$ componentwise as

$$
\begin{array}{r}
{\left[S_{k, i}(\eta, U)\right]_{h, j}:=x_{h}^{\top} P\left(\left(A_{i}+\eta I_{n}\right) x_{j}+B_{i} u_{j}\right)+} \\
\beta_{j}^{\top} P\left(\left(A_{i}+\eta I_{n}\right) x_{h}+B_{i} u_{h}\right),
\end{array}
$$

where $x_{h}, x_{j} \in \mathcal{V}_{k}$. Then $V_{1}$ and $V_{2}$ have the control-sharing property if there exist $\eta>0, \mathcal{M}$-matrices $W_{1}, W_{2}, \ldots, W_{N} \in$ $\mathbb{R}^{s \times s}$ and $U=\left[u_{1}|\ldots| u_{s}\right] \in \mathbb{R}^{m \times s}$ such that (9) holds and the matrices $-S_{k, i}(\eta, U)$ are copositive ${ }^{1}$ for all $k \in[1, r]$ and $i \in[1, N]$.

The condition proposed in Theorem 6 requires the solution of a copositive programming problem. This problem is convex, but still hard to solve. A sufficient condition which can be checked via LP is that the matrices $S_{k, i}(\eta, U)$ have nonpositive elements.

Corollary 1. Under the assumptions of Theorem 6, $V_{1}$ and $V_{2}$ have the control-sharing property if there exist $\eta>0, \mathcal{M}$ matrices $W_{1}, W_{2}, \ldots, W_{N} \in \mathbb{R}^{s \times s}$ and $U \in \mathbb{R}^{m \times s}$ such that (9) holds and the elements (13) of $S_{k, i}(\eta, U)$ are non-positive for all $k \in[1, r]$ and $i \in[1, N]$.

Then, we consider positive definite 0 -symmetric functions $V_{s}: \mathbb{R}^{n} \rightarrow \mathbb{R}_{\geq 0}$ defined as

$$
V_{s}(x):=\max \left\{x^{\top} Q_{k} x \mid k \in[1, s]\right\}
$$

for some $Q_{1}, Q_{2}, \ldots, Q_{s} \succcurlyeq 0$, hence covering the case of symmetric polyhedral functions, truncated ellipsoids and max of quadratics.

Theorem 7. Assume that $V_{1}=V_{s}$ (14) and $V_{2}(x)=x^{\top} P x$ respectively are CLF and QCLF for (6). Then $V_{1}$ and $V_{2}$ have the control-sharing property if there exist $\eta>0, \lambda_{i, j, k} \geq 0$, $K_{k} \in \mathbb{R}^{m \times n}$, for $i=1,2, \ldots, N$, and $j, k=1,2, \ldots, s$, such that

$$
\begin{aligned}
\left(A_{i}+B_{i} K_{k}\right)^{\top} Q_{k} & +Q_{k}\left(A_{i}+B_{i} K_{k}\right) \preccurlyeq \\
& -2 \eta Q_{k}+\sum_{j=1}^{s} \lambda_{i, j, k}\left(Q_{j}-Q_{k}\right)
\end{aligned}
$$

\footnotetext{
${ }^{1} M$ is copositive if $x^{\top} M x \geq 0$ for all nonnegative vectors $x$.
}
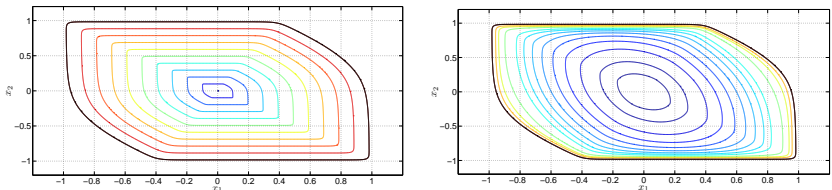

Fig. 2. Level sets of the CLF $V_{1}$ (left) and of a merging function (right) between $V_{1}$ and $V_{2}$ composed via R-functions.

$$
\begin{aligned}
\left(A_{i}+B_{i} K_{k}\right)^{\top} P+ & P\left(A_{i}+B_{i} K_{k}\right) \preccurlyeq \\
& -2 \eta P+\sum_{j=1}^{s} \lambda_{i, j, k}\left(Q_{j}-Q_{k}\right)
\end{aligned}
$$

for all $i \in[1, N], k \in[1, s]$.

Remark 8. Theorem 7 is more general than [25, Theorem 2], because condition (15) relies on a piecewise-linear common controller, rather than a linear common controller as in $[25$, matrix conditions (11)].

\section{THE R-COMPOSITION AS AN EXAMPLE OF MERGING}

We start by considering as an example the simple double integrator system

$$
\dot{x}=\left[\begin{array}{ll}
0 & 1 \\
0 & 0
\end{array}\right] x+\left[\begin{array}{l}
0 \\
1
\end{array}\right] u
$$

with constraints $\|x\|_{\infty} \leq 1,\|u\|_{\infty} \leq 1$.

A typical problem is to choose between a CLF $V_{1}(x)$ assuring a "large" domain of attraction, see Figure 2, or a function which is "locally optimal" in some sense, such as $V_{2}(x)=x^{\top} P x$. In this section, we indeed investigate the "R-composition" proposed in [25], [28] between two homogeneous CLFs, which is shown to be a regular gradienttype merging CLF in the sequel. The main idea is merging the two given functions by a non-homogeneous one which looks like $V_{2}(x)$ close to 0 and like $V_{1}(x)$ far from 0 as in Figure 2 (right). A CLF with such characteristics is a typical example of (regular) gradient-type merging CLF.

The composition consists of the following steps.

1) Define ${ }^{2} R_{1}, R_{2}: \mathbb{R}^{n} \rightarrow \mathbb{R}$ as $R_{i}(x)=1-V_{i}(x), i=1,2$.

2) For fixed $\phi>0$, define $R_{\wedge}: \mathbb{R}^{n} \rightarrow \mathbb{R}$ as $^{34}$

$R_{\wedge}(x):=\rho(\phi)\left(\phi R_{1}(x)+R_{2}(x)-\sqrt{\phi^{2} R_{1}(x)^{2}+R_{2}(x)^{2}}\right)$,

where $\rho(\phi):=\left(\phi+1-\sqrt{\phi^{2}+1}\right)^{-1}$ is the normalization factor [25, Section 2].

3) Define the "R-composition" $V_{\wedge}: \mathbb{R}^{n} \rightarrow \mathbb{R}_{\geq 0}$ as

$$
V_{\wedge}(x):=1-R_{\wedge}(x) .
$$

\footnotetext{
${ }^{2}$ The level set 1 is taken without loss of generality. With this choice we have $R_{i}(x) \geq 0 \Leftrightarrow x \in \mathcal{L}_{V_{i}}$.

${ }^{3}$ For ease of reading, the dependence of $R_{\wedge}$ from $\phi$ is not made explicit in the notation.

${ }^{4}$ All the technical properties of the R-composition presented later on are still true if we consider the more-general definition $R_{\wedge}(x):=$ $\rho_{p}(\phi)\left(\phi R_{1}(x)+R_{2}(x)-\sqrt[2 p]{\left(\phi R_{1}(x)\right)^{2 p}+R_{2}(x)^{2 p}}\right)$, for arbitrary integer $p \geq 1$.
} 
It turns out that [25, Proof of Theorem 1]

$$
\nabla V_{\wedge}(x)=\rho(\phi)\left[\phi c_{1}(\phi, x) \nabla V_{1}(x)+c_{2}(\phi, x) \nabla V_{2}(x)\right],
$$

where $c_{1}, c_{2}: \mathbb{R}_{>0} \times \mathbb{R}^{n} \rightarrow \mathbb{R}_{\geq 0}$ are defined as

$$
\begin{aligned}
c_{1}(\phi, x) & :=1+\frac{-\phi R_{1}(x)}{\sqrt{\phi^{2} R_{1}(x)^{2}+R_{2}(x)^{2}}}, \\
c_{2}(\phi, x) & :=1+\frac{-R_{2}(x)}{\sqrt{\phi^{2} R_{1}(x)^{2}+R_{2}(x)^{2}}} .
\end{aligned}
$$

It follows from the properties of the "R-functions", see Appendix $\mathrm{B}$, that $V_{\wedge}$ is positive definite (Lemma 1), differentiable in int $\mathcal{L}_{V_{\wedge}}$ (Lemma 2), and that $\mathcal{L}_{V_{\wedge}}=\mathcal{L}_{V_{1}} \cap \mathcal{L}_{V_{2}}$ (Lemma 3).

The function $V_{\wedge}$, namely the merging of $V_{1}$ and $V_{2}$, will be used as a candidate CLF later on.

Proposition 5. $V_{\wedge}$ is a gradient-type merging candidate.

We can now show that $V_{\wedge}$ is a regular merging-type candidate with arbitrarily small tolerance.

Proposition 6. Let $\mathcal{L}_{V_{2}} \supset \mathcal{L}_{V_{1}}$. Then for any $\varepsilon>0$ and $\delta \in(0,1)$ there exists $\bar{\phi}>0$ such that for all $\phi \geq \bar{\phi}$ we have that $V_{\wedge}$, with domain $\mathcal{L}_{\left(V_{\wedge} / \delta\right)}$, is a regular gradient-type merging candidate with tolerance $\varepsilon$.

According to Theorem 2 and Theorem 4, if $V_{1}$ and $V_{2}$ are CLFs for (4) and share a constrained control law $\kappa$, then $\kappa$ is admissible as well for $V_{\wedge}$, which turns out to be a CLF for (4) under constraints.

It follows from the proof of Lemma 1 that, independently from $\phi>0$, the unit level set of $V_{\wedge}$ is $\partial \mathcal{L}_{V_{\wedge}}=\{x \in$ $\left.\mathbb{R}^{n} \mid \max \left\{V_{1}(x), V_{2}(x)\right\}=1\right\}$. Conversely, in int $\mathcal{L}_{V_{\wedge}}, \phi$ imposes a trade-off between the shape of the level sets of $V_{1}$ and of $V_{2}$. Namely, in light of [25, Proposition 2], we have $V_{\wedge}(x) \stackrel{\phi \rightarrow \infty}{\longrightarrow} V_{2}(x)$ and $V_{\wedge}(x) \stackrel{\phi \rightarrow 0^{+}}{\longrightarrow} V_{1}(x)$, point-wise in int $\mathcal{L}_{V_{\wedge}}$. Moreover, according to Lemmas 4,5 , 6, we have $\nabla V_{\wedge}(x) \stackrel{\phi \rightarrow \infty}{\longrightarrow} \nabla V_{2}(x)$ and $\nabla V_{\wedge}(x) \stackrel{\phi \rightarrow 0^{+}}{\longrightarrow} \nabla V_{1}(x)$ uniformly on compact subsets of int $\mathcal{L}_{V_{\wedge}}$.

This particular property of fixing the "external" shape, while making the "inner" one "close" to any given choice can be exploited to fix a "large" DoA while achieving "locallyoptimal" closed-loop performances.

Remark 9. We remind that the (smoothed) polyhedral functions of the kind [29], [30], [31], [27], composite quadratics [32] and the convex hull of quadratics [7] are universal classes of homogeneous functions for the stability/stabilizability of LDIs (6). Exploiting Lemma 6, we can merge one of them with any $V_{2}$ (homogeneous of degree 2) to indeed achieve a new class of universal non-homogeneous Lyapunov functions as shown in [33].

\section{A. Controller design under constraints}

We now investigate the existence of a continuous locallyoptimal control under constraints $x \in \mathcal{L}_{V_{1}}$ and $u \in \mathbb{U} \subseteq \mathbb{R}^{m}$ which is closed (possibly compact) and convex. For simplicity, we consider (6) with $B_{i}=B$ for all $i \in[1, N]$. Since the CLF $V_{\wedge}$ is differentiable, in principle, the existence of a stabilizing control law $\kappa$ continuous with the exception of the origin, or including $x=0$ if $V_{\wedge}$ satisfies the small control property ${ }^{5}$, could be proved by using the arguments in [34, Chapters $2-$ 4].

We basically start from $V_{1}$ characterized by a desired, "large", controlled DoA and from $V_{2}$ associated with the desired "locally-optimal" performance. Now, in order to have $\mathcal{L}_{V_{\wedge}}=\mathcal{L}_{V_{1}}$, we preliminary scale $V_{2}$ so that $\mathcal{L}_{V_{2}} \supset \mathcal{L}_{V_{1}}$. In light of Theorem 4, we formulate the control-sharing assumption, which can be checked using the results in Section IV.

Assumption 1. Functions $V_{1}$ and $V_{2}$, homogeneous of degree 2 , have the control-sharing property under constraints $x \in$ $\mathcal{L}_{V_{1}} \subset \mathcal{L}_{V_{2}}$, where $\mathcal{L}_{V_{1}}$ is the "desired" controlled DoA, and $u \in \mathbb{U}$. Associated with $V_{2}$ there is an "optimal" continuous control law $\kappa_{2}: \mathbb{R}^{n} \rightarrow \mathbb{R}^{m}$ such that $\kappa_{2}(x) \in \mathbb{U}$ for all $x$ in a neighborhood of the origin.

We consider the set $\mathcal{U}(x)$ of all admissible controls associated with the merging $\mathrm{CLF} V_{\wedge}$ as

$\mathcal{U}(x):=\left\{u \in \mathbb{U} \mid \max _{i \in[1, N]} \nabla V_{\wedge}(x)\left(A_{i} x+B u\right)+\eta x^{\top} x \leq 0\right\}$

for some $\eta>0$.

For given state $x$, among all the admissible control vectors in $\mathcal{U}(x)$, we take the control $u$ which has smallest distance from the desired one $\kappa_{2}(x)$, namely

$$
\kappa(x):=\arg \min _{v \in \mathcal{U}(x)}\left\|v-\kappa_{2}(x)\right\| .
$$

The above controller only requires the computation of $\nabla V(x)$ in (18) and the solution of a tractable convex optimization problem with decision variable in $\mathbb{R}^{m}$.

The control law $\kappa$ in (18), associated with $V_{\wedge}$, inherits the benefits of both $V_{1}$ and $V_{2}$ according to the following statement.

Proposition 7. Suppose Assumption 1 holds. Then the control law $\kappa(21)$ associated with $V_{\wedge}(17)$ is continuous, satisfies the constraints in $\mathcal{L}_{V_{1}}$, and is locally optimal.

Remark 10. In the case of constrained "linear-quadratic" (LQ) stabilization, the approximate Hamilton-Jacobi-Bellman control $\tilde{\kappa}: \mathcal{L}_{V_{1}} \rightarrow \mathcal{U}(x)$ defined as

$$
\tilde{\kappa}(x):=\arg \min _{v \in \mathcal{U}(x)} \nabla V_{\wedge}(x)(A x+B v)+x^{\top} Q x+v^{\top} R v
$$

has been proposed in [25, Section 5]. An advantage of $\kappa(21)$ over $\tilde{\kappa}$ is that, according to Proposition 7, local optimality is here guaranteed.

\section{B. Illustrative example}

We address the constrained stabilization of a simplified inverted pendulum, whose dynamics is given by the nonlinear differential equation $I \ddot{\theta}(t)=m g l \sin (\theta(t))+\tau(t)$. The goal is the stabilization of $(\theta, \dot{\theta})$ to the origin, under the constraints $|\theta| \leq \frac{\pi}{4},|\dot{\theta}| \leq \frac{\pi}{4}$ and $|\tau| \leq 2$. With notation $x_{1}=\theta$,

${ }^{5} \mathrm{~A}$ CLF $V$ satisfies the small control property if, for $u:=\kappa(x)$, we have that for all $v \in \mathbb{R}_{>0}$ there exists $\epsilon \in \mathbb{R}_{>0}$ so that, whenever $\|x\|<\epsilon$ we have $\|u\|<v[26]$. 

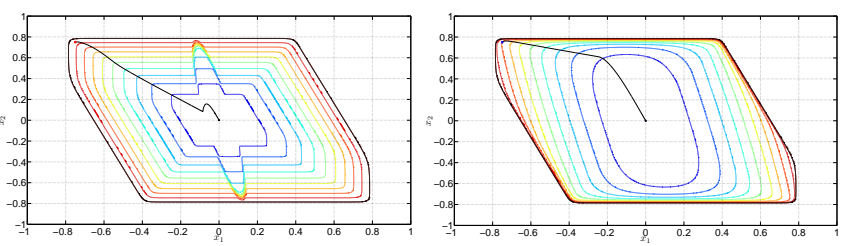

Fig. 3. Left plot: level curves of the uniting CLF [18, (8)-(11)]. Right plot: level curves of the merging CLF $V_{\wedge}(17)$.

$x_{2}=\dot{\theta}=\dot{x}_{1}, u=\tau$ and $w(x):=\left\{\frac{\sin \left(x_{1}\right)}{x_{1}}|| x_{1} \mid \leq \frac{\pi}{4}\right\}$, the following constrained uncertain linear model can be derived.

$$
\dot{x} \in\left[\begin{array}{cc}
0 & 1 \\
a w(x) & 0
\end{array}\right] x+\left[\begin{array}{l}
0 \\
b
\end{array}\right] u,
$$

where $a=(m g l / I), b=(1 / I) ; w(x) \in[0.89,1], w(0)=1$; $\left|x_{1}\right| \leq \pi / 4,\left|x_{2}\right| \leq \pi / 4,|u| \leq 2$. The numerical parameters used in the simulation are $I=0.05, m=0.5, g=9.81$, $l=0.3$.

We adopt the infinite-horizon quadratic performance cost $J(x, u):=\int_{0}^{\infty}\left(\|x(t)\|_{Q}^{2}+\|u(t)\|_{R}^{2}\right) d t$, with weight matrices $Q=I_{2}, R=10$. Let us indeed define the locally-optimal (i.e. for $w \equiv 1$ ) cost function $\bar{V}_{2}(x)=x^{\top} P x$, where $P$ is the unique solution of the Algebraic Riccati Equation. It can be shown that function $\bar{V}_{1}(x)=\|F x\|_{\infty}^{2}$, with $F=$ $\left[\begin{array}{ccc}0 & 1.53 & 4 / \pi \\ 4 / \pi & 0.51 & 0\end{array}\right]^{\top}$, is a PCLF for the constrained LDI (22) and therefore also for the constrained nonlinear system. Then we define the smoothed PCLF $V_{1}(x)=\|F x\|_{40}^{2}$ [27] and we indeed focus on the controlled DoA $\mathcal{L}_{V_{1}}$. Let us also define $V_{2}$ scaling $\bar{V}_{2}$, so that $\mathcal{L}_{V_{2}} \supset \mathcal{L}_{V_{1}}$. Since the LMI condition (7) is satisfied under constraints, $V_{1}$ and $V_{2}$ share a constrained control law in $\mathcal{L}_{V_{1}}$, therefore any gradient-type merging is a CLF. We indeed construct a composite CLF $V_{\wedge}$ with $\phi=10$.

Now, $V_{1}$ has a "large" DoA but it induces a "poor" performance when used with gradient-based controllers of the kind (21). On the other hand, $V_{2}$ is locally optimal, but both gradient-based controllers, for instance (21) with $V_{2}$ in place of $V_{\wedge}$, and the standard LQ regulator yield constraint violations, even in the case with $w \equiv 1$. We notice that $V_{\wedge}$, see Figure 3, with controller (21), inherits the benefits of both $V_{1}$ ("large" DoA under constraints) and $V_{2}$ (local optimality).

From our numerical experience on this example, the merging CLF $V_{\wedge}$ yields better (i.e. in terms of infinite-horizon quadratic performance cost $J$ ) closed-loop performances with respect to the uniting CLF [18, (8)-(11)] when the control control law (21) is employed. This is due to the fact that, unlike the uniting function $[18,(8)-(11)]$, the shape of the merging CLF $V_{\wedge}$ composed via R-functions can be made "close" to the one of $V_{2}$ in the interior of $\mathcal{L}_{V_{1}}$, as shown in Figure 3. Figure 4 shows typical closed-loop state and control trajectories.

For the linearized system (i.e. for $w \equiv 1$ ), our extensive Monte Carlo numerical experiments show that the closed-loop performance is "quite close" to the constrained "global optimal" (obtained via a receding "very-long" horizon controller, under a "fine" system discretization).

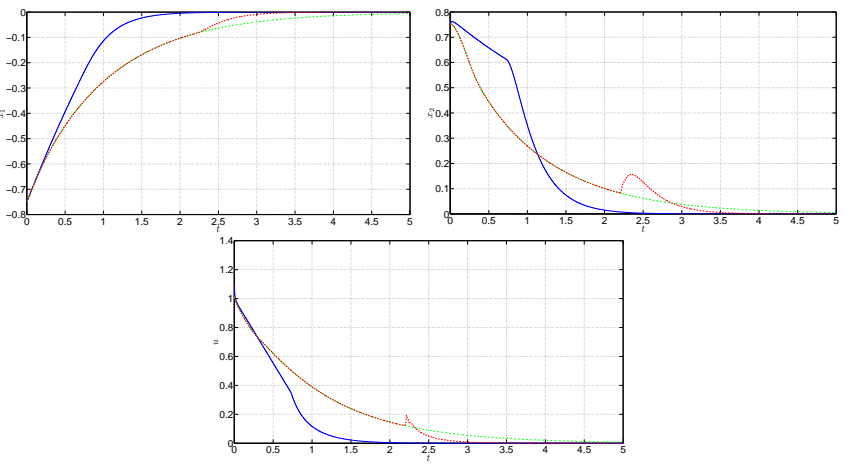

Fig. 4. The control law (21) is associated, respectively, with the uniting CLF [18, (8)-(11)] (dashed lines), the merging CLF $V_{\wedge}$ (17) composed via R-functions (solid lines), and the smoothed polyhedral CLF $V_{1}(x)=\|F x\|_{\infty}^{2}$ (dash-dotted lines). Closed-loop state evolutions: $x_{1}(t)$ (top left), $x_{2}(t)$ (top right), and the control signal $u(t)$ (bottom).

\section{CONCLUSION}

The problem of merging two Lyapunov functions is considered important for several applications, mainly because when concerning constraints, robustness and optimality, a single Lyapunov function is typically suitable for one of these goals, but ineffective for the others.

Previous results show how to combine Lyapunov functions if these share a common control in a suitable region of the state space. For the class of nonlinear control-affine systems, both differential equations and inclusions, we have shown the equivalence between the control-sharing property and the existence of merging control Lyapunov functions.

In order to guarantee the existence of a common control law, linear programs and linear matrix inequalities conditions have been presented for the class of linear differential inclusions.

As an example of merging procedure, a constructive technique based on the R-composition has been given. Further numerical experiments on practical case studies have to be presented. From our experience, our approach is quite close to the constrained global optimality, but no "close form" bounds have been given.

\section{APPENDIX A PROOFS}

\section{A. Proof of Theorem 1}

We have to show that given $\kappa_{1}, \kappa_{2}: \mathbb{R}^{2} \rightarrow \mathbb{R}^{m}$ such that for all $x \in \mathbb{R}^{2}$ we have $\nabla V_{i}(x)\left(A x+B \kappa_{i}(x)\right)<0$, for $i=1,2$, then for all $x \in \mathbb{R}^{2}$ there exists $u \in \mathbb{R}^{m}$ such that the two inequalities $\nabla V_{1}(x)(A x+B u)<0$ and $\nabla V_{2}(x)(A x+B u)<$ 0 can be simultaneously satisfied.

Without any restriction, we assume $m=1$, so that $B \in \mathbb{R}^{2 \times 1}$, otherwise the proof would be trivial. Assume by contradiction that $V_{1}$ and $V_{2}$ do not share a common control, i.e. there exists a point $z \neq 0$ such that the two inequalities (3a)-(3b) are not simultaneously satisfied.

If $z$ and $B$ are aligned, namely $z=\lambda B$ for some $\lambda \neq 0$, we can take $u=-c / \lambda$, for some $c>0$, so that we get

$$
\nabla V_{1}(z)(A z+B u)=\nabla V_{1}(z) A z-c \nabla V_{1}(z) z<0
$$




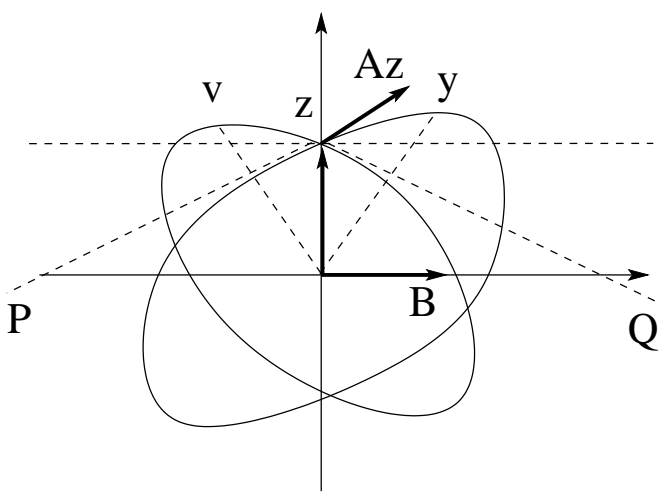

Fig. 5. Geometric construction of the proof of Theorem 1 .

$$
\nabla V_{2}(z)(A z+B u)=\nabla V_{2}(z) A z-c \nabla V_{2}(z) z<0 .
$$

Since $V_{1}$ and $V_{2}$ are convex and positive definite, we have $\nabla V_{1}(z) z>0$ and $\nabla V_{2}(z) z>0$, therefore for $c$ large enough we have (23a)-(23b) simultaneously satisfied.

Let $z$ and $B$ be not aligned and hence consider the state transformation $\hat{x}:=[B \mid z]^{-1} x$, so that $\hat{B}:=[B \mid z]^{-1} B=$ $(1,0)^{\top}$ and $\hat{z}:=[B \mid z]^{-1} z=(0,1)^{\top}$ as in Figure 5. We make this transformation for ease of understanding, so that in the sequel we consider $z=(0,1)^{\top}$ and $B=(1,0)^{\top}$.

Then consider the equation $\dot{z}=(A z+B u)=-\omega z$ in the unknown $u$ and $\omega$, or equivalently $[B \mid z]\left(\begin{array}{c}u \\ \omega\end{array}\right)=-A z$, which has unique solution as $[B \mid z]=I_{2}$. Multiplying both sides by $z^{\top}$ we get $z^{\top} A z+z^{\top} B u=z^{\top} A z=-\omega z^{\top} z$, hence $\omega$ has opposite sign to $z^{\top} A z$.

Therefore if $\omega>0$ then we have $\dot{z}=A z+B u=$ $-\omega z$ so that we simultaneously get $\nabla V_{1}(z)(A z+B u)=$ $-\omega \nabla V_{1}(z) z<0$ and $\nabla V_{2}(z)(A z+B u)=-\omega \nabla V_{2}(z) z<0$.

In the remaining part of the proof, we hence have to consider the case $\omega<0$.

The vector $A z$ must be directed upwards, see Figure 5, so that $z^{\top} A z \geq 0$.

Notice that $\nabla V_{i}(z) B \neq 0$, for $i=1,2$. In fact, let, by contradiction, $\nabla V_{1}(z) B=0$. Then $\nabla V_{1}(z)$ is aligned to $z$ and points upwards, i.e. $\nabla V_{1}(z)=c z$ for some $c>0$. But then $\nabla V_{1}(z)\left(A z+B u_{1}\right)=c z^{\top} A z \geq 0 \forall u_{1} \in \mathbb{R}$, contradicting the assumption that $V_{1}$ is a CLF. Similarly, also $\nabla V_{2}(z) B=0$ would contradict the fact that $V_{2}$ is a CLF.

If $\nabla V_{1}(x) B$ and $\nabla V_{2}(x) B$ have the same sign, then (3a) and (3b) can be simultaneously satisfied for negative $u$ with $|u|$ large enough.

Let $\nabla V_{1}(x) B$ and $\nabla V_{2}(x) B$ have opposite sign. Consider the compact sets $\mathcal{S}_{1}=\left\{x \in \mathbb{R}^{2} \mid V_{1}(x) \leq V_{1}(z)\right\}$ and $\mathcal{S}_{2}=$ $\left\{x \in \mathbb{R}^{2} \mid V_{2}(x) \leq V_{2}(z)\right\}$. The tangent lines to $\mathcal{S}_{1}$ and $\mathcal{S}_{2}$ in $z$ (which is on the boundary of both sets, see lines $P-z$ and $Q-z$ in Figure 5) respectively have positive and negative slope, as an immediate consequence that $\nabla V_{1}(z) B$ and $\nabla V_{2}(z) B$ have opposite signs.

Now let $v$ and $y$ be the "highest" points respectively inside $\mathcal{S}_{1}$ and $\mathcal{S}_{2}$, namely the solutions of the following convex optimization problems: $v:=\arg \max \left\{z^{\top} x \mid x \in \mathcal{S}_{1}\right\}$ and $y:=\arg \max \left\{z^{\top} x \mid x \in \mathcal{S}_{2}\right\}$. Note that $v$ and $y$ are necessarily in the second and in the first quadrant respectively, since the tangent lines in $z$ have opposite slopes. In view of the optimality conditions, we must have that the two gradients are vertical, then aligned with $z: \nabla V_{1}(v)=c_{1} z^{\top}$, $\nabla V_{2}(y)=c_{2} z^{\top}$, for some $c_{1}, c_{2}>0$. Therefore they are orthogonal to $B: \nabla V_{1}(v) B=\nabla V_{2}(y) B=0$.

On the other hand, we assumed that $V_{1}$ and $V_{2}$ are CLFs, i.e. in $v$ and $y$, where the control is "ineffective", we have

$$
\begin{aligned}
& \begin{aligned}
\nabla V_{1}(v)\left(A v+B \kappa_{1}(v)\right) & =\nabla V_{1}(v) A v=c_{1} z^{\top} A v<0 \\
\nabla V_{2}(y)\left(A y+B \kappa_{2}(y)\right) & =\nabla V_{2}(y) A y=c_{2} z^{\top} A y<0,
\end{aligned} \\
& \text { so } z^{\top} A v<0 \text { and } z^{\top} A y<0 .
\end{aligned}
$$

We finally get a contradiction because $z$ is in the cone generated by $v$ and $y$, therefore $z=\alpha v+\beta y$ for some $\alpha, \beta>0$, and $z^{\top} A z=\alpha z^{\top} A v+\beta z^{\top} A y<0$, contradicting the fact that $z^{\top} A z \geq 0$.

\section{B. Proof of Proposition 1}

We show a numerical example for $n=2, m=1, N=2$, in which two QCLFs $V_{1}(x)=x^{\top} P_{1} x$ and $V_{2}(x)=x^{\top} P_{2} x$ do not share a common controller.

Consider (6) with

$$
\begin{gathered}
A_{1}=\left[\begin{array}{rr}
-1.408 & -0.476 \\
0.819 & -1.694
\end{array}\right], A_{2}=\left[\begin{array}{ll}
-0.357 & 1.196 \\
-1.428 & 1.721
\end{array}\right], \\
B_{1}=B_{2}=B=\left[\begin{array}{c}
-1.981 \\
0.600
\end{array}\right] .
\end{gathered}
$$

The eigenvalues of $A_{1}, A_{2}$ respectively are $\{-1.55 \pm i 0.61\}$ and $\{0.68 \pm i 0.79\}$.

Let us consider

$$
P_{1}=\left[\begin{array}{rr}
3.478 & -3.988 \\
-3.988 & 7.825
\end{array}\right], P_{2}=\left[\begin{array}{rr}
4.610 & -18.53 \\
-18.53 & 96.40
\end{array}\right] .
$$

With the linear controllers $\kappa_{1}(x)=K_{1} x$ and $\kappa_{2}(x)=K_{2} x$, being $K_{1}=(0.4815,-0.6934)$ and $K_{2}=(8.310,-42.17)$, we have $\left(A_{j}+B K_{i}\right)^{\top} P_{i}+P_{i}\left(A_{j}+B K_{i}\right) \preccurlyeq-\epsilon_{i} I_{n}, \forall i, j \in$ $\{1,2\}$, with $\epsilon_{1}, \epsilon_{2} \leq 10^{-3}$. Therefore $x^{\top} P_{1} x$ and $x^{\top} P_{2} x$ are CLFs for (6).

Then, we show that for the state $\bar{x}=(-1.813,-0.404)^{\top}$, there cannot exists a common control $u \in \mathbb{R}$, i.e. the following system of equations is not admissible.

$$
\begin{cases}\nabla V_{1}(\bar{x})\left(A_{1} \bar{x}+B u\right)<0, & \nabla V_{1}(\bar{x})\left(A_{2} \bar{x}+B u\right)<0, \\ \nabla V_{2}(\bar{x})\left(A_{1} \bar{x}+B u\right)<0, & \nabla V_{2}(\bar{x})\left(A_{2} \bar{x}+B u\right)<0 .\end{cases}
$$

In fact, we have $\frac{1}{2} \nabla V_{1}(\bar{x}) A_{2} \bar{x}=\bar{x}^{\top} P_{1} A_{2} \bar{x}=6.94$, $\frac{1}{2} \nabla V_{1}(\bar{x}) B=\bar{x}^{\top} P_{1} B=11.74$, therefore $u<-0.59<$ 0 ; however $\frac{1}{2} \nabla V_{2}(\bar{x}) A_{1} \bar{x}=\bar{x}^{\top} P_{2} A_{1} \bar{x}=1.89$ and $\frac{1}{2} \nabla V_{2}(\bar{x}) B=\bar{x}^{\top} P_{2} B=-1.48$, therefore $u>1.28>0$.

\section{Proof of Proposition 2}

We show a numerical example for $n=3$, in which two QCLFs $V_{1}(x)=x^{\top} P_{1} x$ and $V_{2}(x)=x^{\top} P_{2} x$ do not share a common controller.

Consider (5) with

$$
A=\left[\begin{array}{rrr}
-1.990 & -1.135 & -1.063 \\
1.745 & 0.536 & -0.429 \\
-0.794 & -1.243 & -1.813
\end{array}\right], B=\left[\begin{array}{r}
-1.925 \\
-0.342 \\
0.257
\end{array}\right] .
$$

Note that the eigenvalues of $A$ are $\{0.276,-1.772 \pm i 0.114\}$. 
Let us consider $P_{1}=\left[\begin{array}{rrr}35.3372 & 27.5098 & -39.0922 \\ 27.5098 & 21.4164 & -30.4326 \\ -39.0922 & -30.4326 & 43.2484\end{array}\right], P_{2}=$
$\left[\begin{array}{rrr}0.00031 & 0.04321 & -0.01465 \\ 0.04321 & 80.5695 & -39.5654 \\ -0.01465 & -39.5654 & 19.6646\end{array}\right]$.
With the linear controllers $\kappa_{1}(x)=K_{1} x$ and $\kappa_{2}(x)=$ $K_{2} x$, being $K_{1}=(0.5037,0.5799,-0.2013)$ and $K_{2}=$ $(4.5451,4.5697,-0.0669)$, we have $\left(A+B K_{i}\right)^{\top} P_{i}+P_{i}(A+$ $\left.B K_{i}\right) \preccurlyeq-\epsilon_{i} I_{n}$, for $i=1,2$, with $\epsilon_{1}, \epsilon_{2} \leq 10^{-4}$. Therefore $x^{\top} P_{1} x$ and $x^{\top} P_{2} x$ are CLFs.

Then, we show that for the state $\bar{x}=$ $(-0.329,-1.094,-1.537)^{\top}$, there cannot exists a common control $u \in \mathbb{R}$, i.e. the following equations are not simultaneously admissible.

$$
\nabla V_{1}(\bar{x})(A \bar{x}+B u)<0, \quad \nabla V_{2}(\bar{x})(A \bar{x}+B u)<0 .
$$

In fact, $\frac{1}{2} \nabla V_{1}(\bar{x}) A \bar{x}=\bar{x}^{\top} P_{1} A \bar{x}=-31.89, \frac{1}{2} \nabla V_{2}(\bar{x}) A \bar{x}=$ $\bar{x}^{\top} P_{2} A \bar{x}=71.07, \quad \frac{1}{2} \nabla V_{1}(\bar{x}) B=\bar{x}^{\top} P_{1} B=-45.46$, $\frac{1}{2} \nabla V_{2}(\bar{x}) B=\bar{x}^{\top} P_{2} B=12.76$ therefore we get $-31.91-$ $45.46 u<0 \Leftrightarrow u>-0.70 ; 71.07+12.76 u<0 \Leftrightarrow u<$ -5.57 , that clearly is not feasible.

Remark 11. The sets of equations (24) and (25) are not influenced by any scaling of the matrices $P_{i}$, meaning that the set of admissible solutions remains the same for $P_{i} \mapsto \delta_{i} P_{i}$, $\delta_{i}>0, i=1,2$. Such a scaling would influence $\epsilon_{1}, \epsilon_{2}$ in $\left(A_{j}+B K_{i}\right)^{\top} P_{i}+P_{i}\left(A_{j}+B K_{i}\right) \preccurlyeq-\epsilon_{i} I_{n}$ in the following sense. For any $\bar{\epsilon}_{1}, \bar{\epsilon}_{2}>0$, there exist $\delta_{1}, \delta_{2}>0$ such that $\left(A_{j}+B K_{i}\right)^{\top} \delta_{i} P_{i}+\delta_{i} P_{i}\left(A_{j}+B K_{i}\right) \preccurlyeq-\bar{\epsilon}_{i} I_{n}$ for $i=1,2$. That is to say that we cannot run into numerical problems caused by "too small" $\epsilon_{1}, \epsilon_{2}$.

\section{Proof of Theorem 2}

$V$ is a CLF if and only if for any $x \in \mathbb{R}^{n}$ there exists $u \in \mathbb{R}^{m}$ such that $\nabla V(x)(f(x)+g(x) u)<0$. Assume that $V$ is a CLF and let $x$ be fixed. By definition, for any $\gamma_{1}, \gamma_{2} \geq 0$ with $\left(\gamma_{1}, \gamma_{2}\right) \neq(0,0)$, there exists $u \in \mathbb{R}^{m}$ such that $\left(\gamma_{1} \nabla V_{1}(x)+\gamma_{2} \nabla V_{2}(x)\right)(f(x)+g(x) u)<0$, or equivalently for any $\left(\alpha_{1}, \alpha_{2}\right) \in \mathcal{A}:=\left\{(a, b) \in\left(\mathbb{R}_{\geq 0}\right)^{2} \mid a+b=1\right\}$ there exists $u \in \mathbb{R}^{m}$ such that

$$
\left(\alpha_{1} \nabla V_{1}(x)+\alpha_{2} \nabla V_{2}(x)\right)(f(x)+g(x) u)<0 .
$$

Therefore we have

$\max _{\left(\alpha_{1}, \alpha_{2}\right) \in \mathcal{A}} \inf _{u \in \mathbb{R}^{m}}\left(\alpha_{1} \nabla V_{1}(x)+\alpha_{2} \nabla V_{2}(x)\right)(f(x)+g(x) u)<0$.

Since $\mathcal{A}$ is compact and $\mathbb{R}^{m}$ is closed, and the function in (26) is linear in both $\left(\alpha_{1}, \alpha_{2}\right)$ and $u$, we can exchange "max" and "min" [35, Corollary 37.3.2] to get the following equivalent condition.

$$
\begin{gathered}
\max _{\left(\alpha_{1}, \alpha_{2}\right) \in \mathcal{A}} \inf _{u \in \mathbb{R}^{m}}\left(\alpha_{1} \nabla V_{1}(x)+\alpha_{2} \nabla V_{2}(x)\right)(f(x)+g(x) u)= \\
\inf _{u \in \mathbb{R}^{m}} \max _{\left(\alpha_{1}, \alpha_{2}\right) \in \mathcal{A}}\left(\alpha_{1} \nabla V_{1}(x)+\alpha_{2} \nabla V_{2}(x)\right)(f(x)+g(x) u)= \\
\inf _{u \in \mathbb{R}^{m}} \max _{\left(\alpha_{1}, \alpha_{2}\right) \in \mathcal{A}}\left\{\alpha_{1} \nabla V_{1}(x)(f(x)+g(x) u)+\right. \\
\left.\alpha_{2} \nabla V_{2}(x)(f(x)+g(x) u)\right\}<0 \Longleftrightarrow \\
\inf _{u \in \mathbb{R}^{m}} \max \left\{\nabla V_{1}(x)(f(x)+g(x) u),\right. \\
\left.\nabla V_{2}(x)(f(x)+g(x) u)\right\}<0 .
\end{gathered}
$$

The last inequality is equivalent to the existence of a common controller. The result follows as all the considered inequalities are equivalent.

\section{E. Proof of Theorem 3}

We first notice that a regular gradient-type merging always exists, for instance $V(x):=V_{1}(x) V_{1}(x)+\left(1-V_{1}(x)\right) V_{2}(x)$. We also notice that 2) also assumes the existence of a regular control law. Therefore the implication 2) $\Longrightarrow 1$ ) follows immediately.

In view of Theorem 2,3$) \Longrightarrow 2$ ). We then prove 1$) \Longrightarrow 3$ ).

Assume that a regular merging exists, namely that $\left(\gamma_{1}(x) \nabla V_{1}(x)+\gamma_{2}(x) \nabla V_{2}(x)\right)(A x+B u(x))<0$, for some regular control law $u(\cdot)$. Let $p>0$ be the degree of homogeneity (common by assumption) of the CLFs $V_{1}$ and $V_{2}$.

Given a unit vector $v \in \mathbb{R}^{n},\|v\|=1$, consider the ray $\mathcal{R}:=$ $\left\{\lambda v \in \mathbb{R}^{n} \mid \lambda>0\right\}$. Since the functions are homogeneous, their gradients along $\mathcal{R}$ are aligned, namely for all $x=\lambda v$ we have $\nabla V_{1}(x)=\lambda^{p} \nabla V_{1}(v)$ and $\nabla V_{2}(x)=\lambda^{q} \nabla V_{2}(v)$ for some $p, q>0$. Therefore we have

$\left(\gamma_{1}(\lambda v) \lambda^{p} \nabla V_{1}(v)+\gamma_{2}(\lambda v) \lambda^{q} \nabla V_{2}(v)\right)(\lambda A v+B u(\lambda v))<0$, or equivalently (divide by $\gamma_{1}(\lambda v) \lambda^{p}+\gamma_{2}(\lambda v) \lambda^{q}>0$ and by $\lambda>0$ ) to get

$$
\left(\begin{array}{l}
\underbrace{\frac{\gamma_{1}(\lambda v) \lambda^{p}}{\gamma_{1}(\lambda v) \lambda^{p}+\gamma_{2}(\lambda v) \lambda^{q}}}_{=: \alpha_{1}(\lambda)} \nabla V_{1}(v)+ \\
\underbrace{\frac{\gamma_{2}(\lambda v) \lambda^{q}}{\gamma_{1}(\lambda v) \lambda^{p}+\gamma_{2}(\lambda v) \lambda^{q}}}_{=: \alpha_{2}(\lambda)} \nabla V_{2}(v))(A v+B \omega)<0,
\end{array}\right.
$$

where we define $\omega:=u(\lambda v) / \lambda$. Denote by $\bar{\lambda}$ the value of $\lambda$ such that $\bar{\lambda} v \in \partial \mathcal{L}_{V}$, i.e. $V(\bar{\lambda} v)=1$. For all $\lambda \in[0, \bar{\lambda}]$, we have $\alpha_{1}(\lambda)+\alpha_{2}(\lambda)=1$ and $\alpha_{1}(\lambda), \alpha_{2}(\lambda) \geq 0$. Moreover as $\lambda$ goes from 0 to $\bar{\lambda}$, both $\alpha_{1}(\lambda)$ and $\alpha_{2}(\lambda)=1-\alpha_{1}(\lambda)$ assume all values from 0 to 1 , because we have assumed that the merging is regular.

This means that for all $\left(\alpha_{1}, \alpha_{2}\right) \in \mathcal{A}:=\{(a, b) \in$ $\left.\left(\mathbb{R}_{\geq 0}\right)^{2} \quad \mid a+b=1\right\}$ there exists $\omega \in \mathbb{R}^{m}$ such that $\left(\alpha_{1} \nabla V_{1}(v)+\alpha_{2} \nabla V_{2}(v)\right)(A v+B \omega)<0$, i.e.

$$
\max _{\left(\alpha_{1}, \alpha_{2}\right) \in \mathcal{A}} \inf _{\omega \in \mathbb{R}^{m}}\left(\alpha_{1} \nabla V_{1}(v)+\alpha_{2} \nabla V_{2}(v)\right)(A v+B \omega)<0 .
$$

To complete the proof we just need to apply the same minmax argument of the proof of Theorem 2 .

\section{F. Proof of Proposition 3}

We prove the claim by means of an example with $n=m=$ 2. Consider the linear system $\dot{x}=u$, along with the linear controllers $\kappa_{1}(x)=K_{1} x$, with $K_{1}=\left[\begin{array}{cc}-\epsilon & 1 / a \\ -a & -\epsilon\end{array}\right]$, and $\kappa_{2}(x)=$ $K_{2} x$, with $K_{2}=K_{1}^{\top}=\left[\begin{array}{cc}-\epsilon & -a \\ 1 / a & -\epsilon\end{array}\right]$, for some $a, \epsilon>0$. The functions $V_{1}(x)=\frac{1}{2}\left(a x_{1}^{2}+\frac{1}{a} x_{2}^{2}\right), V_{2}(x)=\frac{1}{2}\left(\frac{1}{a} x_{1}^{2}+a x_{2}^{2}\right)$, 
are two QCLFs, respectively with controllers $\kappa_{1}$ and $\kappa_{2}$. In fact, since $\nabla V_{1}(x)=\left(a x_{1}, \frac{1}{a} x_{2}\right), \nabla V_{2}(x)=\left(\frac{1}{a} x_{1}, a x_{2}\right)$, we have $\nabla V_{i}(x)(A x+B u)=-\epsilon V_{i}(x)$, for $i=1,2$.

Take any gradient-type merging candidate $\nabla V(x)=$ $\left(\gamma_{1}(x) \nabla V_{1}(x)+\gamma_{2}(x) \nabla V_{2}(x)\right)$ and $\left\{\gamma_{1}(x)=1, \gamma_{2}(x)=0\right\}$ "far" from the state-space origin and, vice-versa, $\left\{\gamma_{1}(x)=\right.$ $\left.0, \gamma_{2}(x)=1\right\}$ "close" to the origin. Therefore $V$ is such that $\nabla V(x)=\nabla V_{1}(x)$ "far" from the origin and $\nabla V(x)=$ $\nabla V_{2}(x)$ "close" to the origin. The controller $\bar{\kappa}(x)=-\epsilon x$ assures that $V$ a CLF, as $\nabla V(x)(A x+B u)=-\epsilon \nabla V(x) x=$ $-\epsilon\left(\nabla V_{1}(x)+\nabla V_{2}(x)\right) x=-\epsilon\left(a+\frac{1}{a}\right)\left(x_{1}^{2}+x_{2}^{2}\right)$ is negative definite $\forall \epsilon, a>0$.

Note that for $a \gg 1$ the vector $\nabla V_{1}$ is almost "horizontal", while the vector $\nabla V_{2}$ is almost "vertical". Consider the ray (bisector) $\mathcal{R}=\{x=(\xi, \xi), \xi \geq 0\}$. Since $\nabla V$ is continuous, there exists a point $\mathcal{R}$ on the bisector in which $\nabla V$ is aligned to the bisector itself, i.e. there exist $\lambda, \xi \geq 0$ such that $\nabla V(\xi)=\lambda \cdot(\xi, \xi)$. In such a point, with both $\kappa_{1}(\xi)$ and $\kappa_{2}(\xi)$, we have $\nabla V(\xi)\left(A \xi+B \kappa_{i}(\xi)\right)=\lambda\left(-2 \epsilon+\left(\frac{1}{a}-a\right)\right) \xi^{2}$ that is strictly positive for $\epsilon \ll 1, a \gg 1$.

\section{G. Proposition 4}

The proof is similar to the proof of Theorem 2 .

\section{H. Proof of Theorem 4}

The implication $(2) \Rightarrow(1)$ follows from Proposition 4 . To prove the claim $(1) \Rightarrow(2)$ we write $G(x)=g(x)$ to mean that $G$ is single-valued. Fix arbitrary $\gamma_{1}, \gamma_{2}>0$ and define

$$
\bar{f}(x):=\arg \max _{\varphi \in F(x)}\left(\gamma_{1} \nabla V_{1}(x)+\gamma_{2} \nabla V_{2}(x)\right) \varphi .
$$

Now, by assumption we have that

$$
\max _{\varphi \in F(x)}\left(\gamma_{1} \nabla V_{1}(x)+\gamma_{2} \nabla V_{2}(x)\right)(\varphi+g(x) u)<0,
$$

namely that

$$
\begin{aligned}
\max _{\varphi \in F(x)}\left\{\left(\gamma_{1} \nabla V_{1}(x)+\gamma_{2} \nabla V_{2}(x)\right) \varphi\right\}+ \\
\left(\gamma_{1} \nabla V_{1}(x)+\gamma_{2} \nabla V_{2}(x)\right) g(x) u<0 .
\end{aligned}
$$

According to the definition of $\bar{f}$, the first term can be written as $\left(\gamma_{1} \nabla V_{1}(x)+\gamma_{2} \nabla V_{2}(x)\right) \bar{f}(x)$. Finally, we can just follow the proof of Theorem 2 for the nonlinear system $\dot{x}=\bar{f}(x)+$ $g(x) u$.

\section{Proof of Theorem 5}

Necessity of the condition is immediate because if for each $x$ there exists $u(x)$ such that both functions $V_{1}$ and $V_{2}$ are decreasing, then for all vertices $\bar{x}_{k}^{1}$ the condition (12) is satisfied with $u_{k}:=u\left(\bar{x}_{k}^{1}\right)$.

To prove sufficiency we need to show that if the inequality (12) holds for each $\bar{x}_{k}^{1}$, then for each $x$ there exists a control $u$ which is suitable for both $V_{1}$ and $V_{2}$. We borrow ideas from the Gutman and Cwikel piecewise-linear control [3], [14]. Let $x \neq 0$ be arbitrary.

We can always select a subset of $n$ columns of $\bar{X}^{1}$ such that any $x$ is in the simplicial cone with non-empty interior

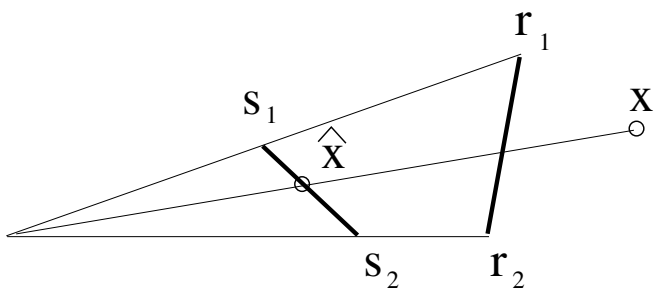

Fig. 6. The cone generated by $R=\left[\begin{array}{ll}r_{1} & r_{2}\end{array}\right]$ and $S=\left[\begin{array}{ll}s_{1} & s_{2}\end{array}\right]$.

$\mathcal{S}=\left\{S \gamma \in \mathbb{R}^{n} \mid \gamma \geq \underline{0}_{n}, \gamma \in \mathbb{R}^{n}\right\}$, for some invertible $S \in \mathbb{R}^{n \times n}$

The choice of these columns corresponds to the choice of the aligned columns of $\bar{X}^{2}$, which we group in an invertible matrix $R \in \mathbb{R}^{n \times n}$, so that we also have $\mathcal{S}=\left\{R \delta \in \mathbb{R}^{n}\right.$ $\left.\delta \geq \underline{0}_{n}, \delta \in \mathbb{R}^{n}\right\}$. We can always choose these columns in such a way no other column (of either $\bar{X}^{1}$ or $\bar{X}^{2}$ ) is inside the cone. Therefore the columns forming $S$ and $R$ are necessarily vectors on the same faces of $\mathcal{L}_{V_{1}}$ and of $\mathcal{L}_{V_{2}}$ respectively. We denote by $P:=\left[u_{k, 1}^{1}, \ldots, u_{k, n}^{1}\right]$ the matrix formed by the control values $u_{k}^{1}$ associated with the columns $\left[x_{k, 1}^{1}|\cdots|\right.$ $\left.x_{k, n}^{1}\right]$ forming $S$. Let

$$
u=P \gamma, \quad \text { where } x=S \gamma, \gamma \geq \underline{0}_{n}
$$

namely, $u=P S^{-1} x$, hence this control is linear in the cone. It is immediate to see that if $x=S_{h}$, i.e. $x$ is one of the generator columns, then $u=P_{h}$.

On the other hand each column of $R$ is aligned with a column of $S$, so we have $R=S D$, for some diagonal $D \in$ $\mathbb{R}^{n \times n}$ having positive diagonal coefficients. Then we associate with each column of $R$ the corresponding control in $P$ scaled accordingly, to form a "control matrix" $Q:=P D$. We can hence define a control constructed as in (28):

$$
u=Q \delta, \quad \text { where } x=R \delta, \delta \geq \underline{0}_{n}
$$

(again linear, i.e. $u=Q R^{-1} x$, inside the cone). We notice that (28) and (29) are exactly the same control vector, where the unique vectors $\delta$ and $\gamma$ are related by $\gamma=D \delta$.

On the vertices $S_{h}$ 's, by assumption, for all $i \in[1, N]$ we have the inclusion

$$
\left[\eta I+A_{i}\right] S_{h}+B_{i} P_{h} \in \mathcal{C}^{1}\left(S_{h}\right) \bigcap \mathcal{C}^{2}\left(S_{h}\right)
$$

Since the tangent cones are scaling-invariant, i.e. $\mathcal{C}^{i}(x)=$ $\mathcal{C}^{i}(\lambda x)$ for all $\lambda>0$, for all $i \in[1, N]$ we also have $\left[\eta I+A_{i}\right] R_{h}+B_{i} Q_{h} \in \mathcal{C}^{1}\left(R_{h}\right) \bigcap \mathcal{C}^{2}\left(R_{h}\right)$.

Since the control $u=P \gamma=Q \delta$ is linear in the cone, we can scale $x$ as $\hat{x}:=x / V_{1}(x)$ which is on the involved face of $\mathcal{L}_{V_{1}}$ (see Figure 6): if the inclusion holds in $\hat{x}$, then it holds also in $x$. Such a face contains the vertices forming $S$. The tangent cone on the face is defined by active constraints which are active also on these vertices, then the tangent cone inside the face includes the tangent cones at the vertices, hence $\left[\eta I+A_{i}\right] S_{h}+B_{i} P_{h} \in \mathcal{C}^{1}\left(S_{h}\right) \subseteq \mathcal{C}^{1}(x)$. Therefore

$$
\left[\eta I+A_{i}\right] x+B_{i} u=\sum_{h=1}^{n} \gamma_{h}\left(\left[\eta I+A_{i}\right] S_{h}+B_{i} P_{h}\right) \in \mathcal{C}^{1}(x) .
$$


Exactly in the same way we can prove the inclusion in $\mathcal{C}^{2}(x)$ using (29) for the control $u$, i.e.

$$
\left[\eta I+A_{i}\right] x+B_{i} u=\sum_{h=1}^{n} \delta_{h}\left(\left[\eta I+A_{i}\right] R_{h}+B_{i} Q_{h}\right) \in \mathcal{C}^{2}(x)
$$

\section{J. Proof of Theorem 6}

The assumption that $V_{1}$ is a PCLF is equivalent to the existence of a piecewise-linear controller that follows from the control vectors $u_{1}, u_{2}, \ldots, u_{s}$ (respectively associated with the vertices $x_{1}, x_{2}, \ldots, x_{s}$ ), namely the columns of $U$, which shows up in (9).

According to the same construction of the proof of Theorem 5 , if $\left\{x_{1}, x_{2}, \ldots, x_{r}\right\}$ are the vertices of a given facet of the polyhedron $\mathcal{L}_{V_{1}}$, together with control vectors $\left\{u_{1}, u_{2}, \ldots, u_{r}\right\}$, then the control vector $\bar{u}(\alpha):=\sum_{h=1}^{r} \alpha_{h} u_{h}$, for $\alpha=$ $\left(\alpha_{1}, \alpha_{2}, \ldots, \alpha_{r}\right) \in \mathcal{A}:=\left\{a \in\left(\mathbb{R}_{\geq 0}\right)^{r} \mid \sum_{h=1}^{r} a_{h}=1\right\}$ is an admissible control for $V_{1}$ in the state point $\bar{x}(\alpha):=$ $\sum_{h=1}^{r} \alpha_{h} x_{h}$.

Therefore it is sufficient to prove that for each facet of the polyhedron $\mathcal{L}_{V_{1}}$, the control $\bar{u}(\alpha)$, parameterized by $\alpha \in \mathcal{A}$, is admissible also for $V_{2}$, i.e. there exists $\eta>0$ such that

$$
\bar{x}(\alpha)^{\top} P\left[\left(A_{i}+\eta I_{n}\right) \bar{x}(\alpha)+B_{i} \bar{u}(\alpha)\right] \leq 0 \quad \forall i \in[1, N] .
$$

Then we can write

$$
\begin{gathered}
\left(\sum_{h=1}^{r} \alpha_{h} x_{h}\right)^{\top} P\left[\left(A_{i}+\eta I_{n}\right)\left(\sum_{h=1}^{r} \alpha_{h} x_{h}\right)+\right. \\
\left.B_{i}\left(\sum_{h=1}^{r} \alpha_{h} u_{h}\right)\right] \leq 0 \quad \forall i \in[1, N] \Longleftrightarrow \\
\sum_{h, j=1}^{r} \alpha_{h} \alpha_{j}\left(x_{h}^{\top} P\left[\left(A_{i}+\eta I_{n}\right) x_{j}+B_{i} u_{j}\right]+\right. \\
\left.x_{j}^{\top} P\left[\left(A_{i}+\eta I_{n}\right) x_{h}+B_{i} u_{h}\right]\right) \leq 0 \quad \forall i \in[1, N] .
\end{gathered}
$$

We get that the left-hand side of the last inequality, namely $\alpha^{\top} S_{, i}(\eta, U) \alpha$, has to be non-positive for $\alpha \in\left(\mathbb{R}_{\geq 0}\right)^{r}$. Therefore the matrices $-S_{k, i}(\eta, U)$, where the subscript $k$ indicates the $k$ th facet, have to be copositive. This is equivalent to the assumption made.

\section{K. Proof of Theorem 7}

For all $k \in[1, s]$, define the sectors $\mathcal{S}_{k}:=\left\{x \in \mathbb{R}^{n} \mid\right.$ $\left.x^{\top} P_{k} x \geq \max _{j} x^{\top} P_{j} x\right\}$, so that we have

$$
\begin{aligned}
& x \in \mathcal{S}_{k} \Longrightarrow x^{\top} P_{k} x \geq x^{\top} P_{j} x \forall j \in[1, s] \Longrightarrow \\
& x^{\top}\left(\sum_{j=1}^{s} \lambda_{i, j, k}\left(P_{j}-P_{k}\right)\right) x \leq 0
\end{aligned}
$$

for any $\lambda_{i, j, k} \geq 0$, where $i \in[1, N], j, k \in[1, s]$.

The matrix inequality condition (15a) is necessary and sufficient for $V_{1}$ to be a CLF for (6) [32], with piecewise-linear controller $\kappa(x):=K(x) x$, where $K(x):=\left\{K_{k}\right.$ if $\left.x \in \mathcal{S}_{k}\right\}$. Then we show that (15b) is sufficient for $\kappa$ to be a valid controller also for $V_{2}$.
Consider $x \in \mathcal{S}_{k}$ and multiply (15b) by $x^{\top}$ on the left and by $x$ on the right, so that

$$
\begin{aligned}
& 2 \nabla V_{2}(x)\left(A_{i}+B_{i} K_{k}\right) x= \\
& \quad x^{\top}\left[\left(A_{i}+B_{i} K_{k}\right)^{\top} P+P\left(A_{i}+B_{i} K_{k}\right)\right] x \leq \\
& -2 \eta \underbrace{x^{\top} P x}_{=V_{2}(x)}+x^{\top}\left(\sum_{j=1}^{s} \lambda_{i, j, k}\left(P_{j}-P_{k}\right)\right) x \quad \forall i \in[1, N] .
\end{aligned}
$$

Therefore, in view of (30), we finally get to $\nabla V_{2}(x)\left(A_{i}+\right.$ $\left.B_{i} K_{k}\right) x \leq-\eta V_{2}(x) \forall i \in[1, N]$.

The proof follows since the choice of sector $\mathcal{S}_{k} \ni x$ has been made arbitrarily.

\section{Proof of Proposition 5}

The proof follows from Lemma 2 as $\nabla V_{\wedge}=$ $\rho\left(\phi c_{1} \nabla V_{1}(x)+c_{2} \nabla V_{2}\right)$, where the functions $c_{1}, c_{2}: \mathbb{R}_{>0} \times$ $\mathbb{R}^{n} \rightarrow \mathbb{R}_{\geq 0}$ defined in (19) are continuous.

\section{Proof of Proposition 6}

As $V_{2}$ has been scaled so that $\mathcal{L}_{V_{2}} \supset \mathcal{L}_{V_{1}}$, we have $\mathcal{L}_{V_{\wedge}}=\mathcal{L}_{V_{1}}$ from Lemma 3 . Let us use the notation $\gamma_{1}(x):=\rho(\phi) \phi c_{1}(\phi, x)$ and $\gamma_{2}(x):=\rho(\phi) c_{2}(\phi, x)$, so that $\nabla V_{\wedge}(x)=\gamma_{1}(x) \nabla V_{1}(x)+\gamma_{2}(x) \nabla V_{2}(x)$, where $\gamma_{1}$ and $\gamma_{2}$ also depend on the parameter $\phi$.

According to the proof of Lemma 4, we have the following fact. For any $\varepsilon>0$ and $\delta>0$ there exists $\phi_{1}>0$ such that for all $\phi \geq \phi_{1}$ we have $\max _{x \in \mathcal{L}_{\left(V_{\wedge} / \delta\right)}} \gamma_{1}(x) \leq \varepsilon$. Analogously, for any $\varepsilon>0$ and $\delta>0$ there exists $\phi_{2}>0$ such that for all $\phi \geq \phi_{2}$ we have $1-\varepsilon \leq \max _{x \in \mathcal{L}_{\left(V_{\wedge} / \delta\right)}} \gamma_{1}(x) \leq 1$. The proof is complete if we take $\bar{\phi}:=\max \left\{\phi_{1}, \phi_{2}\right\}$, so that for all $\phi \geq \bar{\phi}$ and $x \in \mathcal{L}_{\left(V_{\wedge} / \delta\right)}$ we have $\gamma_{1}(x) \in[0, \varepsilon]$ and $\gamma_{2}(x) \in[1-\varepsilon, 1]$.

\section{N. Proof of Proposition 7}

According to Theorem $4, V_{\wedge}$ is a CLF in $\mathcal{L}_{V_{\wedge}}$. Moreover, it follows from [25, Proposition 1] that $V_{\wedge}$ grows quadratically, i.e. $\min \left\{\bar{V}_{1}(x), \bar{V}_{2}(x)\right\} \leq V_{\wedge}(x) \leq \max \left\{\bar{V}_{1}(x), \bar{V}_{2}(x)\right\} \forall x \in$ $\mathbb{R}^{n}$. Therefore for some $\eta>0$, we have that for all $x \in \mathcal{L}_{V_{\wedge}}$ there exists $u \in \mathbb{R}^{m}$ such that $\max _{i \in[1, N]} \nabla V_{\wedge}(x)\left(A_{i} x+B u\right) \leq-\eta x^{\top} x$.

We notice that the optimization problem (21) follows from the minimal selection control

$$
\begin{aligned}
m(x):=\arg \min \left\{\begin{array}{l}
\|v\| \mid \max _{i \in[1, N]} \nabla V_{\wedge}(x) A_{i} x+ \\
\left.\nabla V_{\wedge}(x) B\left(v+\kappa_{2}(x)\right) \leq-\eta x^{\top} x\right\},
\end{array}\right.
\end{aligned}
$$

which is known to be continuous [34, Section 4.2]. Hence the optimal solution of (21) can be written as $\kappa(x):=m(x)+$ $\kappa_{2}(x)$, which is the sum of two continuous functions.

In the following, we prove that $\kappa_{2}$ is an admissible control for $V_{\wedge}$ in a neighborhood of the origin. This will also imply that $V_{\wedge}$ satisfies the small control property.

According to Lemma 4, we have the following property. For any $\epsilon>0$ and $\sigma \in(0,1)$ there exists $\bar{\phi}>0$ such 
that $\phi \geq \bar{\phi}$ implies that $\nabla V_{\wedge}(x)=\nabla \bar{V}_{2}(x)+v(x)^{\top}$, with $\max _{x \in \mathcal{L}_{(V \wedge / \sigma)}}\|v(x)\| \leq \epsilon$. Therefore we can write

$$
\begin{gathered}
\max _{i \in[1, N]} \nabla V_{\wedge}(x)\left(A_{i} x+B \kappa_{2}(x)\right)= \\
\max _{i \in[1, N]} \nabla V_{2}(x)\left(A_{i} x+B \kappa_{2}(x)\right)+v(x)^{\top}\left(A_{i} x+B \kappa_{2}(x)\right) \leq \\
\max _{i \in[1, N]} \nabla V_{2}(x)\left(A_{i} x+B \kappa_{2}(x)\right)+ \\
\max _{i \in[1, N]} v(x)^{\top}\left(A_{i} x+B \kappa_{2}(x)\right) . \quad \text { (31) }
\end{gathered}
$$

We notice that there exists $\eta, \sigma_{2}>0$ such that $\max _{i \in[1, N]} \nabla V_{\wedge}(x)\left(A_{i} x+B \kappa_{2}(x)\right) \leq-2 \eta x^{\top} x$ for all $x$ in the compact set $\mathcal{L}_{\left(V_{2} / \sigma_{2}\right)}$. Therefore we choose $\sigma$ so that $\left\{x \in \mathbb{R}^{n} \mid V_{\wedge}(x) \leq \sigma\right\} \subseteq\left\{x \in \mathbb{R}^{n} \mid V_{2}(x) \leq \sigma_{2}\right\}$, namely as $\sigma:=\max \left\{c \in[0,1] \mid \mathcal{L}_{\left(V_{\wedge} / c\right)} \subseteq \mathcal{L}_{\left(V_{2} / \sigma_{2}\right)}\right\}$.

We can now choose $\epsilon \geq\|v(x)\|$ such that $\max _{x \in \mathcal{L}_{(V \wedge / \sigma)}}\left\{\max _{i \in[1, N]} v(x)^{\top}\left(A_{i} x+B \kappa_{2}(x)\right)-\eta x^{\top} x\right\} \leq 0$. Therefore, using the above inequality in (31), we get that $\kappa_{2}$ is an admissible control for $V_{\wedge}$ in a neighborhood of the origin, i.e. $\max _{i \in[1, N]} \nabla V_{\wedge}(x)\left(A_{i} x+B \kappa_{2}(x)\right) \leq-\eta x^{\top} x$. This means that for all $x \in \mathcal{L}_{\left(V_{\wedge} / \sigma\right)}$, the constraint $v \in \mathcal{U}(x)$ in (21) is not active and therefore $\kappa(x)=\kappa_{2}(x)$ is locally optimal. Moreover, we also get that the control law $\kappa$ is continuous also at the origin.

\section{APPENDIX B}

\section{TECHNICAL PROPERTIES OF THE R-COMPOSITION}

Lemma 1. $V_{\wedge}$ is positive definite.

Proof: At the origin we have $V_{1}(0)=V_{2}(0)=0 \Longleftrightarrow$ $R_{1}(0)=R_{2}(0)=1$. Therefore, from $(16), R_{\wedge}(0)=1$ and hence $V_{\wedge}(0)=1-R_{\wedge}(0)=0$. Conversely, $V_{\wedge}(\bar{x})=0 \Leftrightarrow$ $R_{\wedge}(\bar{x})=1$. From [25, Proposition 1], we have $1=R_{\wedge}(\bar{x}) \leq$ $\max \left\{R_{1}(\bar{x}), R_{2}(\bar{x})\right\}$. Since $R_{1}(x) \leq 1$ and $R_{2}(x) \leq 1$ by construction, we have that $R_{1}(\bar{x})=1$ or $R_{2}(\bar{x})=1$ (or both). Say $R_{1}(\bar{x})=1$. Therefore $R_{1}(\bar{x})=1 \Leftrightarrow V_{1}(\bar{x})=0 \Leftrightarrow \bar{x}=0$.

Lemma 2. Assume that $V_{1}$ and $V_{2}$ are differentiable respectively in $\mathcal{L}_{V_{1}}$ and $\mathcal{L}_{V_{2}}$. Then $V_{\wedge}$ is differentiable in int $\mathcal{L}_{V_{\wedge}}$.

Proof: The proof immediately follows from (18) since $\phi>0$ is fixed and functions $c_{i}(\phi, x), i=1,2$, are continuous whenever $R_{1}(x)$ and $R_{2}(x)$ are not simultaneously 0 , i.e. in $\operatorname{int} \mathcal{L}_{V_{\wedge}}$.

For ease of notation, in the following proofs, let us denote $V_{1}(x), V_{2}(x), R_{1}(x), R_{2}(x), c_{1}(\phi, x), c_{2}(\phi, x)$ without the explicit dependence on their arguments.

Lemma 3. $\mathcal{L}_{V_{\wedge}}=\mathcal{L}_{V_{1}} \cap \mathcal{L}_{V_{2}}$.

Proof: According to [25, Lemma 1], we have $R_{\wedge}>$ $0 \Longleftrightarrow\left\{R_{1}>0\right.$ and $\left.R_{2}>0\right\}$; moreover, from (16), $R_{\wedge}=0 \Longleftrightarrow\left\{R_{1}=0\right.$ or $\left.R_{2}=0\right\}$. Now by construction $V_{i}=1-R_{i}, i \in\{1,2\}$, and $V_{\wedge}=1-R_{\wedge}$, therefore $V_{\wedge}<1 \Longleftrightarrow\left\{V_{1}<1\right.$ and $\left.V_{2}<1\right\}$, and $V_{\wedge}=1 \Longleftrightarrow\left\{V_{1}=\right.$ 1 or $\left.V_{2}=1\right\}$, i.e. $\mathcal{L}_{V_{\wedge}}=\mathcal{L}_{V_{1}} \cap \mathcal{L}_{V_{2}}$.

Preprint submitted to IEEE Transactions on Automatic Control. Received: August 19, 2013 02:29:18 PST Copyright (c) 2013 IEEE. Personal use is permitted. For any other purposes, permission must be obtained from the IEEE by emailing pubs-permissions@ieee.org.
Lemma 4. $\nabla V_{\wedge}$ converges to $\nabla V_{2}$ uniformly on compact subsets of int $\mathcal{L}_{V_{\wedge}}$, as $\phi \rightarrow \infty$. Namely, for any $\delta \in(0,1)$ we have $\lim _{\phi \rightarrow \infty} \max _{x \in \mathcal{L}_{\left(V_{\wedge} / \delta\right)}}\left\|\nabla V_{\wedge}(x)-\nabla V_{2}(x)\right\|=0$.

Proof: First we have

$$
\begin{aligned}
\lim _{\phi \rightarrow \infty} \rho(\phi)=\lim _{\phi \rightarrow \infty} \frac{1}{\phi+1-\sqrt{\phi^{2}+1}}= \\
\lim _{\phi \rightarrow \infty} \frac{\phi+1+\sqrt{\phi^{2}+1}}{2 \phi}=1 .
\end{aligned}
$$

Then

$$
\begin{gathered}
\lim _{\phi \rightarrow \infty} \phi c_{1}=\lim _{\phi \rightarrow \infty} \phi\left(1+\frac{-\phi R_{1}}{\sqrt{\phi^{2} R_{1}^{2}+R_{2}^{2}}}\right)= \\
\lim _{\phi \rightarrow \infty} \frac{R_{2}^{2}}{\phi R_{1}^{2}+R_{2}^{2} / \phi+R_{1} \sqrt{\phi^{2} R_{1}^{2}+R_{2}^{2}}} \leq \\
\lim _{\phi \rightarrow \infty} \frac{1}{2 \phi R_{1}^{2}} \leq \lim _{\phi \rightarrow \infty} \frac{1}{2 \phi(1-\delta)^{2}}=0 .
\end{gathered}
$$

The last inequality holds uniformly as $R_{1}(x) \geq 1-\delta>0$ whenever $x \in \mathcal{L}_{\left(V_{\wedge} / \delta\right)}=\left\{y \in \mathbb{R}^{n} \mid V_{\wedge}(y) \leq \delta\right\}$. Then we can also write

$$
\begin{gathered}
\lim _{\phi \rightarrow \infty} c_{2}=\lim _{\phi \rightarrow \infty}\left(1+\frac{-R_{2}}{\sqrt{\phi^{2} R_{1}^{2}+R_{2}^{2}}}\right)= \\
\lim _{\phi \rightarrow \infty} \frac{\sqrt{\phi^{2} R_{1}^{2}+R_{2}^{2}}-R_{2}}{\sqrt{\phi^{2} R_{1}^{2}+R_{2}^{2}}}= \\
\lim _{\phi \rightarrow \infty} \frac{R_{1}^{2}}{R_{1}^{2}+R_{2}^{2} / \phi+\left(R_{2} / \phi\right) \sqrt{R_{1}^{2}+R_{2}^{2} / \phi^{2}}}=1 .
\end{gathered}
$$

Therefore, combining (32), (33) and (34), we get $\lim _{\phi \rightarrow \infty} \nabla V_{\wedge}(x) \quad=\quad \lim _{\phi \rightarrow \infty} \rho(\phi)\left(\phi c_{1}(\phi, x) \nabla V_{1}(x) \quad+\right.$ $\left.c_{2}(\phi, x) \nabla V_{2}(x)\right)=\nabla V_{2}(x)$ uniformly on compact subsets of the kind $\mathcal{L}_{\left(V_{\wedge} / \delta\right)}$.

Lemma 5. $\nabla V_{\wedge}$ converges to $\nabla V_{1}$ uniformly on compact subsets of int $\mathcal{L}_{V_{\wedge}}$, as $\phi \rightarrow 0^{+}$. Namely, for any $\delta \in(0,1)$ we have $\lim _{\phi \rightarrow 0^{+}} \max _{x \in \mathcal{L}_{\left(V_{\wedge} / \delta\right)}}\left\|\nabla V_{\wedge}(x)-\nabla V_{1}(x)\right\|=0$.

Proof: Since $\nabla V_{\wedge}=\rho(\phi)\left[\phi c_{1} \nabla V_{1}+c_{2} \nabla V_{2}\right]$, we have to prove that for any $\delta \in(0,1)$ we have $\lim _{\phi \rightarrow 0^{+}} \rho(\phi) \phi c_{1}(x)=1$ and $\lim _{\phi \rightarrow 0^{+}} \rho(\phi) c_{2}(\phi, x)=0$ for all $x \in \mathcal{L}_{\left(V_{\wedge} / \delta\right)}$.

Similarly to (32) and (33) we have that

$$
\begin{gathered}
\lim _{\phi \rightarrow 0^{+}} \rho(\phi) \phi c_{1}= \\
\lim _{\phi \rightarrow 0^{+}} \frac{\phi}{\phi+1-\sqrt{\phi^{2}+1}}\left(1+\frac{-\phi R_{1}}{\sqrt{\phi^{2} R_{1}^{2}+R_{2}^{2}}}\right)= \\
\lim _{\phi \rightarrow 0^{+}} \frac{\phi+1-\sqrt{\phi^{2}+1}}{2 \phi} \cdot \frac{\phi R_{2}^{2}}{\phi^{2} R_{1}^{2}+R_{2}^{2}+\phi R_{1} \sqrt{\phi^{2} R_{1}^{2}+R_{2}^{2}}} \\
=1 . \quad \text { (35) }
\end{gathered}
$$

The last equality holds uniformly as $R_{1}(x) \geq 1-\delta>0$ and $R_{2}(x) \geq 1-\delta>0$ (both the numerator and the denominator are indeed strictly positive) whenever $x \in \mathcal{L}_{\left(V_{\wedge} / \delta\right)}=\{y \in$ 
$\left.\mathbb{R}^{n} \mid V_{\wedge}(y) \leq \delta\right\}$. Then we can also write $\lim _{\phi \rightarrow 0^{+}} \rho(\phi) c_{2}$ as

$$
\begin{gathered}
\lim _{\phi \rightarrow 0^{+}} \frac{1}{\phi+1-\sqrt{\phi^{2}+1}}\left(1+\frac{-R_{2}}{\sqrt{\phi^{2} R_{1}^{2}+R_{2}^{2}}}\right)= \\
\lim _{\phi \rightarrow 0^{+}} \frac{\phi+1+\sqrt{\phi^{2}+1}}{2 \phi} \cdot \frac{\sqrt{\phi^{2} R_{1}^{2}+R_{2}^{2}}-R_{2}}{\sqrt{\phi^{2} R_{1}^{2}+R_{2}^{2}}}= \\
\lim _{\phi \rightarrow 0^{+}} \frac{\phi+1+\sqrt{\phi^{2}+1}}{2} \cdot \frac{\phi R_{1}^{2}}{\left(\sqrt{\phi^{2} R_{1}^{2}+R_{2}^{2}}+R_{2}\right) \sqrt{\phi^{2} R_{1}^{2}+R_{2}^{2}}} \\
=0 .
\end{gathered}
$$

Since $R_{1}(x), R_{2}(x) \geq 1-\delta>0$, the denominator is strictly positive and hence the last equality holds uniformly. Therefore, from (35) and (36) we get $\lim _{\phi \rightarrow 0^{+}} \rho(\phi)\left(\phi c_{1}(\phi, x) \nabla V_{1}(x)+\right.$ $\left.c_{2}(\phi, x) \nabla V_{2}(x)\right)=\nabla V_{1}(x)$ uniformly on compact subsets of the kind $\mathcal{L}_{\left(V_{\wedge} / \delta\right)}$.

Lemma 6. Assume $\mathcal{L}_{V_{2}} \supset \mathcal{L}_{V_{1}}$. Then $\nabla V_{\wedge}$ converges to $\nabla V_{1}$ uniformly on $\mathcal{L}_{V_{1}}$ as $\phi \rightarrow 0^{+}$, i.e.

$$
\lim _{\phi \rightarrow 0^{+}} \max _{x \in \mathcal{L}_{V_{\wedge}}}\left\|\nabla V_{\wedge}(x)-\nabla V_{1}(x)\right\|=0 .
$$

Proof: We first notice that, as $\mathcal{L}_{V_{2}} \supset \mathcal{L}_{V_{1}}$, we have $\mathcal{L}_{V_{\wedge}}=$ $\mathcal{L}_{V_{1}}$ in view of Lemma 3. Then we can use the same proof of Lemma 5 if we notice that $R_{2}(x)$ is strictly positive in $\mathcal{L}_{V_{\wedge}}$ because $\mathcal{L}_{V_{2}} \supset \mathcal{L}_{V_{1}}=\mathcal{L}_{V_{\wedge}}$. In fact, $R_{2}(x)>0$ implies that both the numerator and the denominator of (35), and also the denominator of (36), are strictly positive for all $x \in \mathcal{L}_{V_{\wedge}}$.

\section{REFERENCES}

[1] F. Schweppe, Uncertain dynamic systems. Englewood Cliff, N.J.: Prentice Hall, 1973.

[2] P. Gutman and P. Hagander, "A new design of constrained controllers for linear systems," IEEE Trans. on Automatic Control, vol. 30, no. 1 , pp. 22-33, 1985.

[3] P. Gutman and M. Cwikel, "Admissible sets and feedback control for discrete-time linear dynamical systems with bounded controls and states," IEEE Trans. on Automatic Control, vol. 31, no. 4, pp. 373-376, 1986.

[4] S. Keerthi and E. Gilbert, "Computation of minimum-time feedback control laws for discrete-time systems with state-control constraints," IEEE Trans. on Automatic Control, vol. 32, no. 5, pp. 432-435, 1987.

[5] F. Blanchini and A. Megretski, "Robust state feedback control of LTV systems: nonlinear is better than linear," IEEE Trans. on Automatic Control, vol. 44, no. 4, pp. 802-807, 1999.

[6] F. Blanchini and S. Miani, "Constrained stabilization via smooth Lyapunov functions," Systems \& Control Letters, vol. 35, pp. 155-163, 1998.

[7] T. Hu and Z. Lin, "Composite quadratic Lyapunov functions for constrained control systems," IEEE Trans. on Automatic Control, vol. 48, no. 3, pp. 440-450, 2003.

[8] B. O'Dell and E. Misawa, "Semi-ellipsoidal controlled invariant sets for constrained linear systems," Journal of Dynamic Systems, Measurement and Control, vol. 124, pp. 98-103, 2002.

[9] T. Thibodeau, W. Tong, and T. Hu, "Set invariance and performance analysis of linear systems via truncated ellipsoids," Automatica, vol. 45, pp. 2046-2051, 2009.

[10] A. Balestrino, E. Crisostomi, S. Grammatico, and A. Caiti, "Stabilization of constrained linear systems via smoothed truncated ellipsoids," in Proc. of the IFAC World Congress, Milan, Italy, 2011.

[11] G. Chesi, A. Garulli, A. Tesi, and A. Vicino, "Homogeneous Lyapunov functions for systems with structured uncertainties," Automatica, vol. 39, no. 6, pp. 1027-1035, 2003.

[12] - Homogeneous polynomial forms for robustness analysis of uncertain systems. Springer, 2009.
[13] T. Hu and Z. Lin, Control of systems with actuator saturation. Boston, MA: Birkhäuser, 2001

[14] F. Blanchini and S. Miani, Set-theoretic methods in control, 1st ed. Birkhäuser, 2007.

[15] G. Wredenhagen and P. Belanger, "Piecewise-linear LQ control for systems with input constraint," Automatica, vol. 30, no. 3, pp. 403-416, 1994.

[16] A. Benzaouia and A. Baddou, "Piecewise linear constrained control for continuous-time systems," IEEE Trans. on Automatic Control, vol. 44, no. 7, pp. 1477-1481, 1999.

[17] V. Andrieu and C. Prieur, "Uniting two Lyapunov functions for affine systems," in Proc. of the IEEE Conf. on Decision and Control, Cancun, Mexico, 2008, pp. 622-627.

[18] _ - "Uniting two Lyapunov functions for affine systems," IEEE Trans. on Automatic Control, vol. 55, no. 8, pp. 1923-1927, 2010.

[19] C. Prieur and Praly, "Uniting local and global controllers," in Proc. of the IEEE Conf. on Decision and Control, Phoenix, Arizona (USA), 1999, pp. 1214-1219.

[20] C. Prieur, "Uniting local and global controllers with robustness to vanishing noise," Mathematics of Control, Signals and Systems, vol. 14, pp. 143-172, 2001

[21] H. Nguyen, P. Gutman, S. Olaru, and M. Hovd, "Implicit improved vertex control for uncertain, time-varying linear discrete-time systems with state and control constraints," Automatica, 2013.

[22] F. Clarke, "Lyapunov functions and discontinuous stabilizing feedback," Annual reviews in control, vol. 35, pp. 13-33, 2011.

[23] A. Balestrino, A. Caiti, E. Crisostomi, and S. Grammatico, "Stabilizability of linear differential inclusions via R-functions," in Proc. of the IFAC Symposium on Nonlinear Control Systems, Bologna, Italy, 2010.

[24] A. Balestrino, E. Crisostomi, and A. Caiti, "Logical composition of Lyapunov functions," International Journal of Control, vol. 84, no. 3, pp. 563-573, 2011.

[25] A. Balestrino, A. Caiti, and S. Grammatico, "A new class of Lyapunov functions for the constrained stabilization of linear systems," Automatica, vol. 48, no. 11, pp. 2951-2955, 2012.

[26] E. Sontag, Mathematical Control Theory. Springer, 1998.

[27] F. Blanchini and S. Miani, "A new class of universal Lyapunov functions for the control of uncertain linear systems," IEEE Trans. on Automatic Control, vol. 44, no. 3, pp. 641-647, 1999.

[28] A. Balestrino, A. Caiti, and S. Grammatico, "Multivariable constrained process control via Lyapunov R-functions," Journal of Process Control, vol. 22, no. 9, pp. 1762-1772, 2012.

[29] R. Brayton and C. Tong, "Constructive stability and asymptotic stability of dynamical systems," IEEE Trans. on Circuits and Systems, vol. 27, no. 11, pp. 1121-1130, 1980 .

[30] A. Molchanov and E. Pyatnitskii, "Lyapunov functions specifying necessary and sufficient conditions of absolute stability of nonlinear nonstationary control systems. Parts I, II, III.” Automatica and Remote Control, vol. 47, no. 3,4,5, pp. 344-354, 443-451, 620-630, 1986.

[31] F. Blanchini, "Nonquadratic Lyapunov functions for robust control," Automatica, vol. 31, no. 3, pp. 451-461, 1995.

[32] T. Hu and F. Blanchini, "Non-conservative matrix inequality conditions for stability/stabilizability of linear differential inclusions," Automatica, vol. 46, pp. 190-196, 2010.

[33] S. Grammatico, F. Blanchini, and A. Caiti, "A universal class of non-homogeneous control Lyapunov functions for linear differential inclusions," in Proc. of the IEEE European Control Conference, Zurich, Switzerland, 2013.

[34] R. Freeman and P. Kokotović, Robust nonlinear control design. Boston: Birkhäuser, 1996.

[35] R. Rockafellar, Convex Analysis. Princeton, New Yersey: Princeton University Press, 1970. 


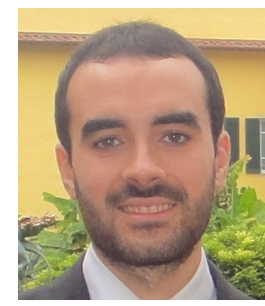

Sergio Grammatico was born in Marsala, Italy, in 1987. He received the B.Sc., M.Sc. and Ph.D. degrees in Automation Engineering from the University of Pisa, Italy, respectively in 2008, 2009, 2013. He also received a M.Sc. degree in engineering from the SantAnna School of Advanced Studies, Pisa, Italy, in 2011. He visited the Department of Mathematics at the University of Hawai'i at Manoa in 2010 and 2011, and the Department of Electrical and Computer Engineering at the University of California Santa Barbara in 2012. He is currently postdoctoral fellow at the Automatic Control Laboratory, ETH Zurich, Switzerland. His research interests include robust Lyapunov-based control and stochastic control systems.

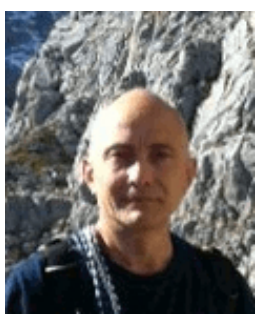

Franco Blanchini was born on 29 December 1959, in Legnano (Italy). He is Director of the Laboratory of System Dynamics of the University of Udine. He has been involved in the organization of international events in particular he was Program Vice-Chairman of the conference Joint CDC-ECC 2005, Seville, Spain; Program Vice-Chairman for the Conference CDC-2008, Cancun, Mexico, Program Chairman of the Conference ROCOND, Aalborg, Denmark, June 2012 and Program Vice-Chairman of the Conference CDC-2013, Florence, Italy, December 2013. He is co-author of the book "Set theoretic methods in control", Birkhauser. He is the recipient of 2001 ASME Oil \& Gas Application Committee Best Paper Award as a co-author of the article "Experimental evaluation of a High-Gain Control for Compressor Surge Instability". He is the recipient of the 2002 IFAC prize survey paper award as author or the article "Set Invariance in Control-a survey", Automatica, November, 1999. He was nominated Senior Member IEEE in 2003. He has been Associate Editor for Automatica from 1996 to 2006. He is Associate Editor for IEEE Transactions on Automatic Control since 2011 .

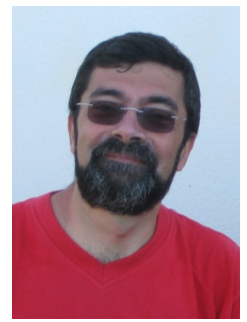

Andrea Caiti was born in Naples, Italy, in 1963 $\mathrm{He}$ received the "Laurea" degree ("cum laude") in electronic engineering from the University of Genova, Genoa, Italy, in 1988. After working as a Staff Scientist at the North Atlantic Treaty Organization Supreme Allied Commander Atlantic Undersea Research Center, La Spezia, Italy, he was with the Department of Electrical Systems and Automation, University of Pisa, in 1996, as an Assistant Professor. In 1998, he was an Associate Professor at the "Dipartimento di Ingegneria dellInformazione", University of Siena, Siena, Italy. Since 2001, he has been with the University of Pisa, where he currently is a Full Professor of Systems Theory and Automatic Control. $\mathrm{He}$ is the Director of the Interuniversity Center of Integrated Systems for the Marine Environment. He is a member of the IEEE Control Systems and IEEE Oceanic Engineering Societies. 